\title{
EPHB2 carried on small extracellular vesicles induces tumor angiogenesis via activation of ephrin reverse signaling
}

\author{
Shinya Sato, ${ }^{1}$ Suhas Vasaikar, ${ }^{2}$ Adel Eskaros, ${ }^{3}$ Young Kim, ${ }^{4}$ James S. Lewis, ${ }^{3}$ Bing Zhang, ${ }^{2}$ \\ Andries Zijlstra, ${ }^{3}$ and Alissa M. Weaver ${ }^{1,3}$ \\ 'Department of Cell and Developmental Biology, Vanderbilt University School of Medicine, Nashville, Tennessee, USA \\ 2Department of Molecular and Human Cenetics, Baylor College of Medicine, Houston, Texas, USA. ${ }^{3}$ Department of \\ Pathology, Microbiology and Immunology, and ${ }^{4}$ Department of Otolaryngology, Vanderbilt University Medical Center, \\ Nashville, Tennessee, USA.
}

\begin{abstract}
Angiogenesis is a key process that allows nutrient uptake and cellular trafficking and is coopted in cancer to enable tumor growth and metastasis. Recently, extracellular vesicles (EVs) have been shown to promote angiogenesis; however, it is unclear what unique features EVs contribute to the process. Here, we studied the role of EVs derived from head and neck squamous cell carcinoma (HNSCC) in driving tumor angiogenesis. Small EVs (SEVs), in the size range of exosomes (50-150 $\mathrm{nm}$ ), induced angiogenesis both in vitro and in vivo. Proteomic analysis of HNSCC SEVs revealed the cell-to-cell signaling receptor ephrin type B receptor 2 (EPHB2) as a promising candidate cargo to promote angiogenesis. Analysis of patient data further identified EPHB2 overexpression in HNSCC tumors to be associated with poor patient prognosis and tumor angiogenesis, especially in the context of overexpression of the exosome secretion regulator cortactin. Functional experiments revealed that EPHB2 expression in SEVs regulated angiogenesis both in vitro and in vivo and that EPHB2 carried by SEVs stimulates ephrin-B reverse signaling, inducing STAT3 phosphorylation. A STAT3 inhibitor greatly reduced SEV-induced angiogenesis. These data suggest a model in which EVs uniquely promote angiogenesis by transporting Eph transmembrane receptors to nonadjacent endothelial cells to induce ephrin reverse signaling.
\end{abstract}

Conflict of interest: The authors have declared that no conflict of interest exists.

Copyright: (c) 2019, American Society for Clinical Investigation.

Submitted: August 12, 2019

Accepted: October 23, 2019

Published: December 5, 2019

Reference information: /CI Insight. 2019;4(23):e132447.

https://doi.org/10.1172/jci.

insight.132447.

\section{Introduction}

Head and neck squamous cell carcinoma (HNSCC) is an invasive cancer that originates in the oral cavity, larynx, and pharynx, in association with alcohol and tobacco use or exposure to human papillomavirus (1-3). While much of the morbidity of HNSCC is caused by local invasion into nerve and vascular tissues of the neck, mortality is driven primarily by metastasis to regional lymph nodes and distant organs (4-7). A key event that facilitates this metastasis is tumor-associated lymph and blood vessel formation (Figure 1A).

Extracellular vesicles (EVs), including exosomes and other small EVs (SEVs) and larger EVs (LEVs) such as microvesicles, are secreted from cells and mediate cell-to-cell communication via protein, lipid, and nucleic acid cargoes (8). EVs are key mediators of cellular functions, such as survival, proliferation, motility, and apoptosis. Recently, many reports have shown that tumor-derived EVs play a large role in tumor progression (9). Many of these functions are due to paracrine and distant signaling to noncancer cells, including induction of cancer-associated fibroblasts, regulation of tumor immunity, and premetastatic niche formation.

Among the paracrine activities, a key reported function of tumor EVs is angiogenesis (10-14). Tumor-derived EVs may also promote lymphangiogenesis $(15,16)$. Despite the number of studies, implicating both RNA $(11,17,18)$ and protein $(10,19,20)$ cargoes, a clear and universal mechanism has not emerged for the apparently critical role of EVs in angiogenesis. It is also not clear whether the same mechanisms will be used for different types of blood vessels or by different tumor types.

Current angiogenesis therapy focuses on soluble secreted molecules, especially VEGF. However, despite the use of anti-VEGF therapy in some cancers, as well as in "wet" age-related macular degeneration (21-25), its utility has been more limited than was originally anticipated (26-28). Therefore, identifying unique mechanisms of angiogenesis is of interest both biologically and therapeutically. Since EVs 
constitute a fundamentally different form of carrier, transporting either internal cytoplasmic cargoes or transmembrane or lipid-linked surface molecules, EV-induced angiogenesis is likely to represent a distinct mode of action from VEGF and other soluble proangiogenesis mediators.

In this study, we investigated the role of EVs released from HNSCC cells on angiogenesis and lymphangiogenesis (Figure 1A). In vivo tumor-associated angiogenesis correlated with the in vitro SEV production rate of several HNSCC cell lines. Furthermore, SEVs purified from HNSCC cells induced angiogenesis, both in vitro and in vivo. Proteomic analysis of SEVs purified from a panel of HNSCC cell lines revealed ephrin-type receptors as candidate angiogenic protein cargoes. Blocking and genetic inhibition experiments validated ephrin type B receptor 2 (EPHB2) as a key SEV cargo that promotes HNSCC-mediated angiogenesis both in vitro and in vivo. Mechanistic experiments indicate that SEV-induced ephrin-B reverse signaling through STAT3 is critical for EV-induced HNSCC angiogenesis.

\section{Results}

Cellular SEV production correlates with HNSCC tumor-induced angiogenesis. To investigate the relationship between EVs and tumor angiogenesis, we correlated the rate of in vitro SEV release by 5 HNSCC cell lines with the capacity of those same cell lines to promote in vivo angiogenesis (Figure 1). SEVs were purified by a cushion density gradient method (29) in order to minimize EV aggregation and enhance EV separation from protein aggregates. EV number, size, marker status, and morphology were characterized by nanoparticle tracking, Western blot, and transmission electron microscopy analyses in accordance with current guidelines (ref. 30 and Supplemental Figure 1, A-C; supplemental material available online with this article; https://doi.org/10.1172/jci.insight.132447DS1). For tumor induction, human (SCC61, OSC19, Detroit 562) and mouse (MOC1, MOC2) HNSCC cell lines were injected orthotopically into the tongues of nude or syngeneic mice. The tumors were harvested, fixed, and immunostained for the endothelial cell marker CD31. Analysis of CD31 area/tumor area revealed that Detroit 562 and MOC2 tumors had significantly higher blood vessel density than tumors derived from the other cell lines (Figure 1, B and C). Detroit 562 and MOC2 cells also exhibited higher rates of in vitro SEV secretion (Figure 1D). Comparison of the association between tumor blood vessel density and SEV secretion rate across all the cell lines by linear regression revealed a strong correlation (Figure $1 \mathrm{E}, R^{2}=0.78$ ).

Purified HNSCC SEVs promote endothelial tube formation. To directly test the angiogenesis-promoting effects of HNSCC-derived SEVs, we performed an in vitro tube-formation assay. HUVECs were treated for 12 hours with conditioned medium or EVs purified from 2 different human HNSCC cell lines, OSC19 and Detroit 562 , before obtaining phase contrast images for analysis of total tube length and number of junctions. Compared with control media, conditioned medium from HNSCC cells significantly increased endothelial tube network formation. By contrast, conditioned medium that was depleted of EVs by ultracentrifugation for 2 hours at 100,000 $\mathrm{g}$ did not increase tube formation over that of control media (Figure 1F). Finally, the addition of purified HNSCC SEVs to the control medium induced formation of a significantly more extensive tube network (Figure 1F). We tested 2 concentrations of SEVs, (a) $5 \times 10^{7}$, which we estimate to be approximately the number of SEVs contained in the $400 \mu \mathrm{L}$ of OSC19 conditioned medium used in the assay, and (b) $1 \times 10^{8}$, which had similar efficacy for both Detroit 562 and OSC19 EVs. In contrast, LEVs at the same concentrations did not affect tube formation (Supplemental Figure 2A). Purified SEVs from 2 other HNSCC cell lines also induced HUVEC tube formation, suggesting that this is a common property of HNSCC EVs (Supplemental Figure 2B). To examine the effects of HNSCC-derived EVs on lymphangiogenesis, we performed the same assay using lymphatic endothelial cells (HMVEC-dLyAds). HNSCC conditioned medium and HNSCC-derived SEVs - but not EV-depleted conditioned medium - facilitated tube network and junction formation in HMVEC-dLyAds similarly to that of HUVECs (Supplemental Figure 2C).

Proteomics and transcriptomics analysis identifies EPHB2 as a candidate angiogenic EV cargo. To determine whether HNSCC SEVs carry angiogenic proteins, we performed proteomic analysis of SEVs purified from 3 different human HNSCC cell lines. One of the data sets was published previously for SEVs from SCC61 cells (31). In addition, we performed proteomics analyses on SEVs purified by cushion density gradient ultracentrifugation from OSC19 and Detroit 562 cells, identifying — respectively — 1016 and 1237 unique proteins (Supplemental Tables 1 and 2). From these 3 data sets, we identified a number of proteins that have been shown to regulate angiogenesis and/or lymphangiogenesis (Table 1; refs. 32-52). Notably, multiple ephrin and ephrin receptor (Eph) family proteins were detected, suggesting that this class of proteins could drive angiogenesis in HNSCC tumors. Furthermore, EPHB2 and EPHA2 were detected in all 3 HNSCC SEV samples (Table 1). 
A

Angiogenesis-mediated event
Lymphangiogenesis-mediated event

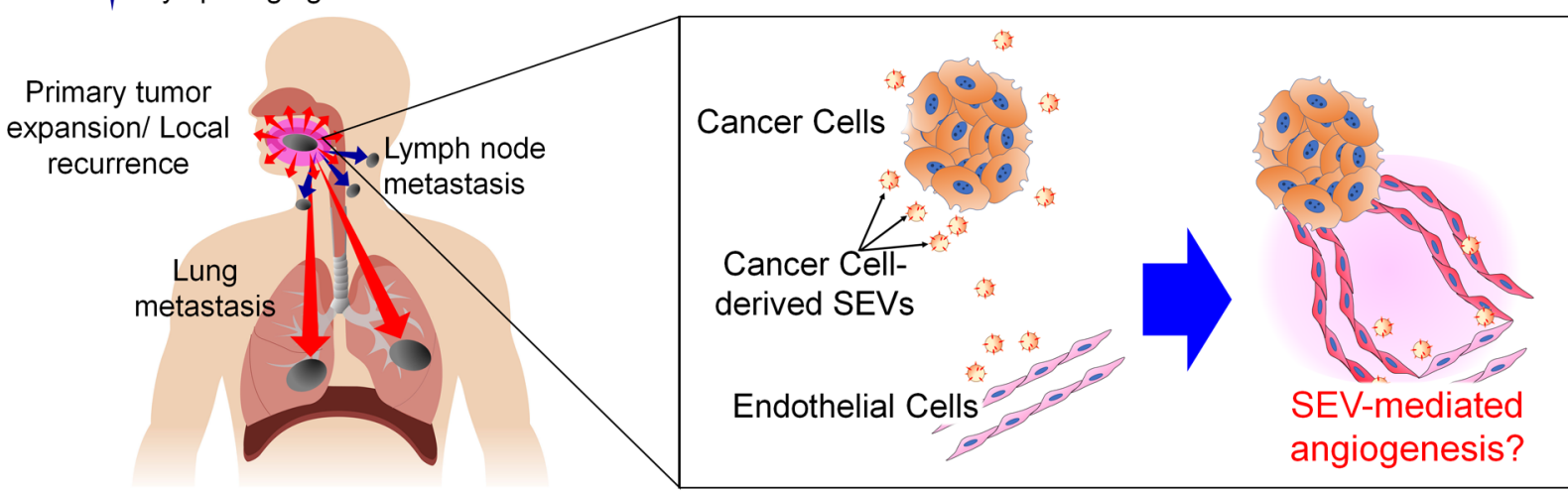

B

Human-HNSCC

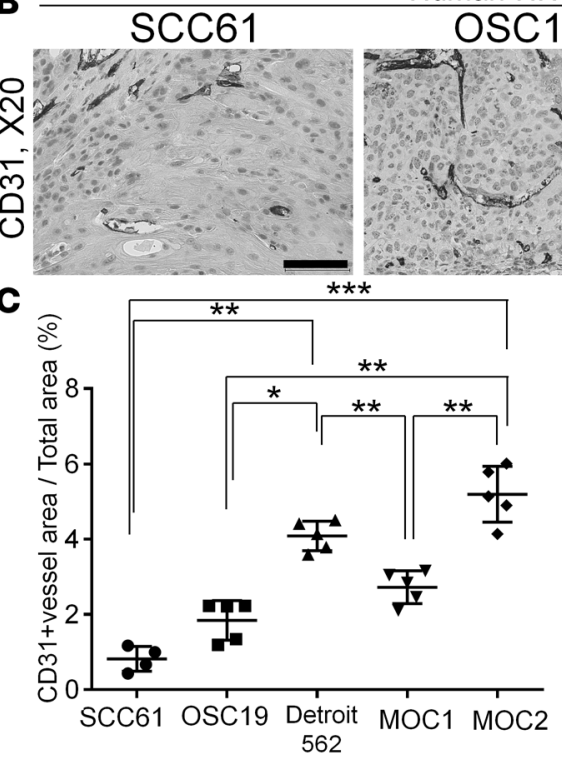

OSC19

Detroit 562



D

$\mathbf{F}$
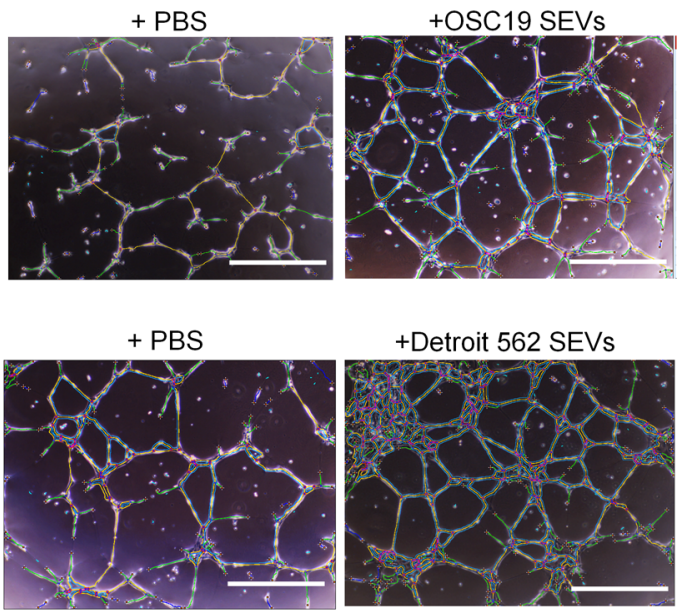

+Detroit 562 SEVs

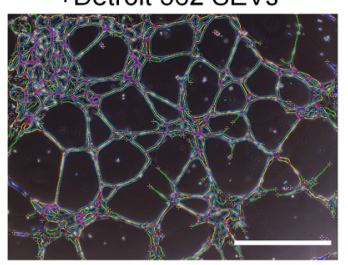

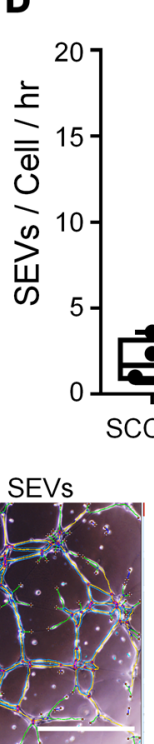

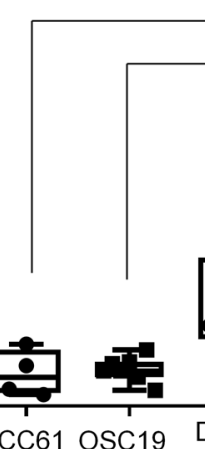

**
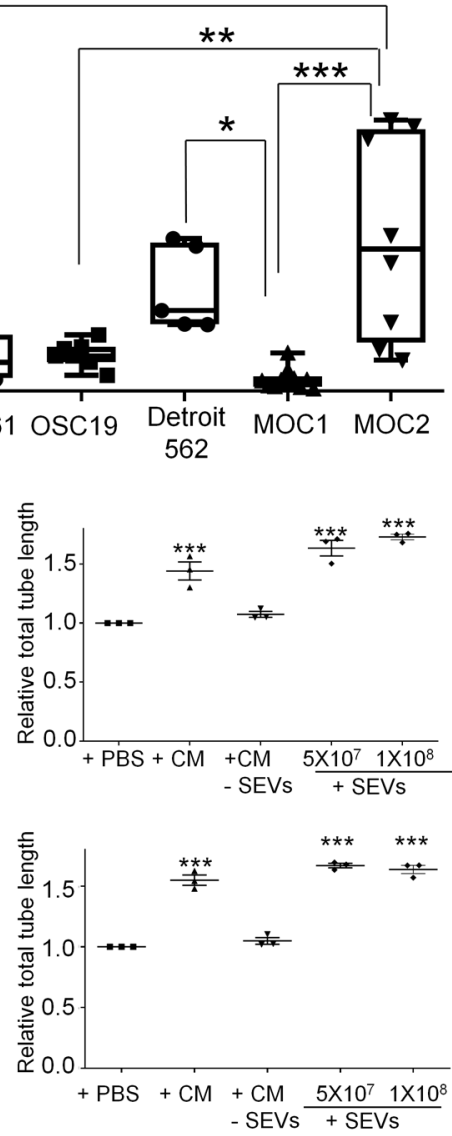

Mouse-HNSCC MOC1 MOC2

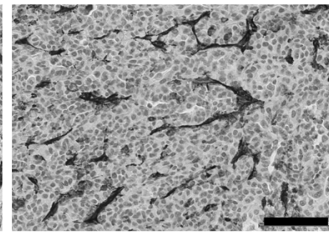

E
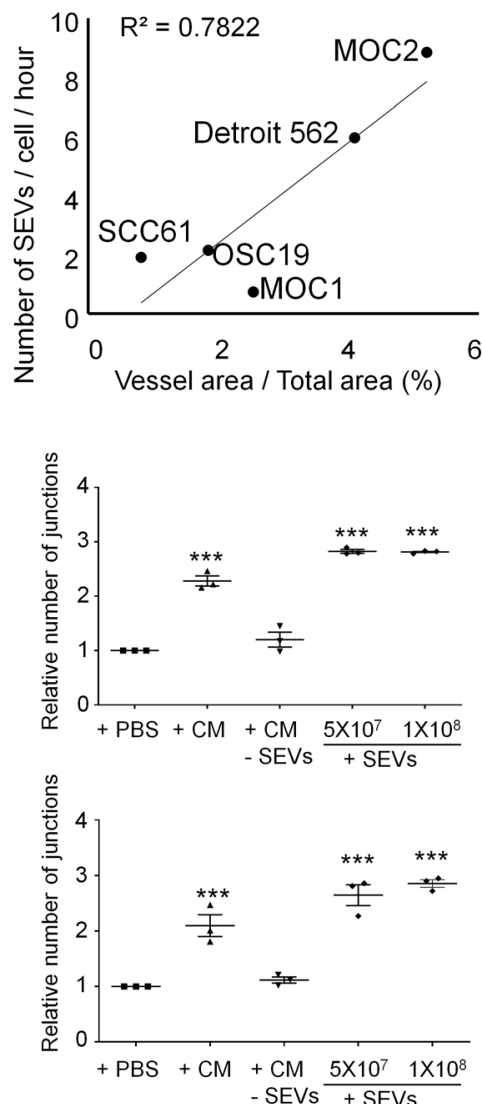

Figure 1. Small extracellular vesicles (SEVs) derived from HNSCC cells drive tumor angiogenesis. (A) Hypothesis for study. EVs mediate angiogenesis and lymphangiogenesis to respectively drive distant and locoregional metastasis. (B) Representative images of tongue tumors derived from SCC61, 0SC19, Detroit 562, MOC1, and MOC2 cells stained for CD31 (black, identifies endothelial cells). SCC61, $n=4$; OSC19, Detroit 562, MOC1, and MOC2, $n=5$. Ten images for each tumor. Scale bar: $100 \mu \mathrm{m}$. (C) Plot of CD31+ vessel area per total tumor area in tongue tumors. SCC61, $n=4$; 0SC19, Detroit 562, MOC1, and MOC2, $n$ $=5$. Total tumor area and CD31-stained area were calculated using Image). (D) SEV secretion rate of cell lines, calculated from nanoparticle tracking analysis of purified vesicles obtained from a known final number of cells over 48 hours. SCC61, $n=4$; OSC19, $n=7$; Detroit 562, $n=5 ;$ MOC1, $n=11 ;$ and MOC2, $n=8$. (E) Linear regression models were performed to analyze relationship between SEV secretion rates and blood vessel density in tumors for various cell lines. 
Adjusted $R^{2}$ of the linear regression model $=0.7822$. (F) HUVECs were incubated for 12 hours in serum-free media plus PBS (control), conditioned media of cancer cells (CM), CM ultracentrifuged to remove SEVs (CM-SEV), or serum free media plus purified cancer cell SEVs $\left(5 \times 10^{7}\right.$ and $\left.1 \times 10^{8}\right)$. (Left) Representative images of HUVEC cells cultured with PBS (control) or OSC19/Detroit 562 SEVs $\left(5 \times 10^{7}\right)$. Imagej outlining of vessels for automated analysis is shown. Scale bar: $500 \mu \mathrm{m}$. (Right) Quantification of relative total tube length and junction numbers for the indicated groups. Plots show the average of $\geq 3$ technical replicates per condition for each $n$ value from $\geq 3$ independent experiments. For $\mathbf{C}, \mathbf{D}$, and $\mathbf{F}$, box-and-whisker plots show median and 25 th-75th percentile. Tukey-Kramer method was used in $\mathbf{C}$ and $\mathbf{D}$, and Dunnett's method was used in $\mathbf{F}$ for statistical analysis. ${ }^{*} P<0.05 ;{ }^{* *} P<0.01 ;{ }^{* * *} P<0.001$.

To prioritize our results, we analyzed publicly available RNA sequencing (RNA-Seq) data for HNSCC patient tumors in the TCGA database to determine whether RNA expression levels of EPHB2 or EPHA2 correlate with patient outcome. This analysis revealed that overexpression of EPHB2 - but not EPHA2 - significantly correlates with decreased survival of HNSCC patients (Figure 2A). EPHB2 is also an interesting cargo, since it can promote ephrin-B reverse signaling $(53,54)$, and studies in genetically engineered mouse models have shown that ephrin-B reverse signaling plays a critical role in both developmental and tumor angiogenesis $(32,55,56)$. In addition, further analysis of the TCGA RNA-Seq data using gene set enrichment analysis (GSEA) revealed that the hallmark angiogenesis gene set is closely correlated with EPHB2 mRNA expression in HNSCC patient tumors (Figure 2B).

We previously showed that the HNSCC-overexpressed protein cortactin promotes exosome secretion (31). Cortactin overexpression had been previously found in small data sets to correlate with poor patient prognosis (57-59). Analysis using the TCGA RNA-Seq data set confirmed that cortactin overexpression significantly correlates with decreased survival of HNSCC patients (Figure 2C). Based on these data, we hypothesized that key exosome cargoes might synergize with cortactin overexpression to drive poor patient prognosis. Indeed, analysis of TCGA RNA-Seq data revealed that co-overexpression of cortactin with EPHB2 further stratified patients into a subset with greatly decreased survival, compared with patient tumors with overexpression of only cortactin, only EPHB2, or no overexpression of either gene (Figure 2D). Conversely, analysis of the relationship between cortactin and EPHB2 expression with the hallmark angiogenesis gene set revealed that only EPHB2 expression correlated with expression of genes in that gene set (Figure 2D and Supplemental Table 3). These data are consistent with cortactin as a general regulator of exosome secretion and EPHB2 as a specific angiogenic cargo. Immunofluorescent analysis of a tissue microarray with HNSCC samples from 103 patients further revealed that EPHB2 and cortactin co-overexpression is closely related to regional lymph node and distant organ metastasis, especially for undifferentiated tumors (Figure 2E and Supplemental Figure 3). Based on these data, we focused on EPHB2 as a promising candidate to test for its role in SEV-mediated angiogenesis.

EPHB2 in SEVS promotes endothelial tube formation. In Western blot analyses, EPHB2 protein was expressed in SEVs from multiple human HNSCC cell lines (Figure 3A). As it was expressed at the highest levels in OSC19 cells, we focused on this cell line for most further mechanistic studies. Consistent with the ability of OSC19 SEVs, but not LEVs, to induce tube formation, we detected very little EPHB2 expression in LEVs in comparison with SEVs (Figure 3A). EPHB2 can induce angiogenesis by binding to B-type ephrins on endothelial cells and inducing ephrin reverse signaling, a process that is thought to require cell-to-cell contacts $(32,55)$. We hypothesized that SEVs could substitute for neighboring cells and induce ephrin reverse signaling at a distance. To test this possibility, we examined phosphorylation of ephrin-B in HUVECs after incubation with OSC19 SEVs. As a positive control, we added recombinant EPHB2 (Fc-EPHB2). As shown in Figure 3B, both Fc-EPHB2 and purified OSC19 SEVs induced ephrin-B phosphorylation in HUVECs, compared with no-treatment control. To test the role of SEV-associated EPHB2 in angiogenesis, we blocked the ability of EPHB2 to bind to ephrin-B ligands on cells by preincubating the OSC19 SEVs with recombinant ephrin-B2 protein (Fc-ephrin-B2) for 10 minutes before adding the mixture to endothelial cells (Figure 3C). Compared with control SEVs, uptake of SEVs preincubated with Fc-ephrin-B2 by HUVEC cells was decreased (Supplemental Figure 4), suggesting that EPHB2-ephrin-B interactions mediate binding of a subset of SEVs with cells. Likewise, preincubation of Fc-ephrin-B2 with SEVs reduced their ability to induce HUVEC tube formation in a dose-dependent manner (Figure 3D). A similar result was observed using SEVs purified from Detroit 562 cells (Figure 3D). Recombinant Fc-ephrin-B2 protein alone had only a small effect in the opposite direction at the highest concentration.

We also tested the effect of SEVs purified from EPHB2-knockdown (EPHB2-KD) or EPHB2-overexpressed (EPHB2-OE) OSC19 cells on angiogenesis. Similar to the results with Fc-ephrin-B2 blocking of EPHB2 on SEVs, tube formation of HUVECs treated with EPHB2-KD SEVs was significantly 
Table 1. Angiogenesis-related proteins in human HNSCC-derived SEVs

\begin{tabular}{|c|c|c|c|c|c|}
\hline \multicolumn{6}{|l|}{ Transmembrane } \\
\hline Protein name & Symbol & $\begin{array}{l}\text { OSC19-derived } \\
\text { SEVs }\end{array}$ & $\begin{array}{l}\text { SCC61-derived } \\
\text { SEVs }\end{array}$ & $\begin{array}{c}\text { Detroit 562-derived } \\
\text { SEVs }\end{array}$ & Refs. \\
\hline Ephrin-B2 & EFNB2 & + & - & - & 32,33 \\
\hline Ephrin type-A receptor 2 & EPHA2 & + & + & + & 36,38 \\
\hline Ephrin type-B receptor 3 & EPHB3 & + & + & - & 34 \\
\hline Matrix metalloproteinase-14 & MMP14 & + & + & - & 45 \\
\hline Hepatocyte growth factor receptor & MET & + & + & + & 45,46 \\
\hline \multicolumn{6}{|l|}{ Membrane binding-cytoplasmic } \\
\hline Protein name & Symbol & $\begin{array}{l}\text { OSC19-derived } \\
\text { SEVs }\end{array}$ & $\begin{array}{l}\text { SCC61-derived } \\
\text { SEVs }\end{array}$ & $\begin{array}{c}\text { Detroit 562-derived } \\
\text { SEVs }\end{array}$ & Refs. \\
\hline Annexin A2 & ANXA2 & + & + & + & 10,51 \\
\hline \multicolumn{6}{|l|}{ Soluble secreted } \\
\hline Protein name & Symbol & $\begin{array}{l}\text { OSC19-derived } \\
\text { SEVs }\end{array}$ & $\begin{array}{l}\text { SCC61-derived } \\
\text { SEVs }\end{array}$ & $\begin{array}{c}\text { Detroit 562-derived } \\
\text { SEVs }\end{array}$ & Refs. \\
\hline VEGF A & VEGFA & - & + & - & 75,77 \\
\hline VEGF C & VEGFC & - & + & - & 77,78 \\
\hline Insulin-like growth factor-binding protein 3 & IGFBP3 & - & + & - & 78 \\
\hline
\end{tabular}

reduced compared with those treated with control SEVs (Figure 4, A and B). Conversely, EPHB2OE SEVs increased HUVEC tube formation (Figure 4, C and D). The enhanced tube formation by EPHB2-OE SEVs was statistically significant compared with control SEVs when a lower number of SEVs $\left(2.5 \times 10^{7}\right)$ was used, as opposed to the higher number $\left(5 \times 10^{7}\right)$ used in the other tube-formation assays. These data indicate that both the number and EPHB2 cargo content of SEVs impact endothelial tube formation.

HNSCC-derived EVs induce in vivo angiogenesis. To test the role of EV-delivered EPHB2 in in vivo angiogenesis, we performed a Matrigel plug assay (60-62) in which SEVs purified from OSC19 cells were mixed with growth factor reduced Matrigel and implanted s.c. in nude mice. After 21 days, the plugs were harvested and analyzed for blood vessel content (Figure 4E). Quantitation of CD31+ vessel area/image area in the plugs showed that control EVs induced blood vessel formation. By contrast, in the absence of EVs or in the presence of EPHB2-KD or Fc-ephrin-B2-pretreated EVs, very few blood vessels were formed (Figure 4E). We verified that the Matrigel plugs indeed contained blood vessels by $\mathrm{H} \& \mathrm{E}$ staining, as well as costaining of some samples for both CD31 and hemoglobin (Supplemental Figure 5A).

To determine whether EPHB2 expression in HNSCC tumors regulates angiogenesis, we injected OSC19 cells into the tongues of nude mice. While there was no effect of EPHB2 expression on tumor size, the vessel density in EPHB2-OE OSC19 tumors was significantly increased compared with control and EPHB2-KD tumors (Figure 4F). EPHB2-KD did not lead to reduced angiogenesis in OSC19 tumors, possibly due to the relatively low basal level of angiogenesis in this cell line (Figure 1E). Indeed, when we assessed angiogenesis in Detroit 562 tumors that have higher baseline levels of angiogenesis and higher SEV secretion rates (Figure 1E), EPHB2-KD reduced angiogenesis (Supplemental Figure 5B). In addition, EPHB2-OE in Detroit 562 cells led to enhanced tumor blood vessel density (Supplemental Figure 5B). Although OSC19 is not a metastatic cell line in this model, we analyzed lymph nodes from these mice. We observed 1 mouse with lymph node metastasis in the EPHB2-OE tumor group, but we observed no metastasis from the other groups (0/10 for SCR, 0/10 for KD, and 1/10 for OE). For the Detroit 562 cells, KD or OE of EPHB2, respectively, decreased and increased the percent of mice with lymph node metastases; however, the differences were not statistically significant (Supplemental Figure 5C). 
A
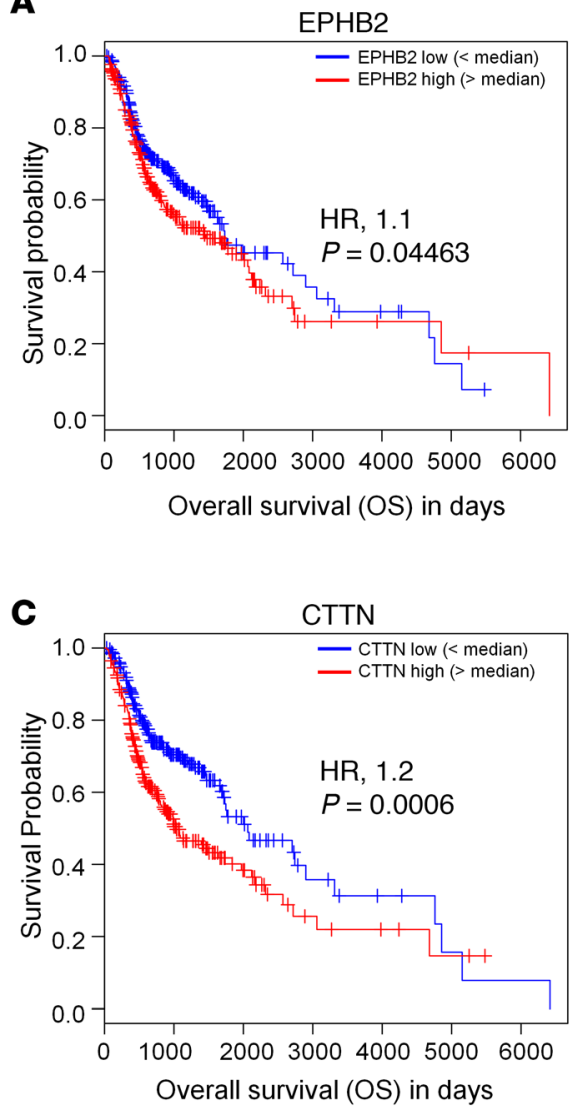
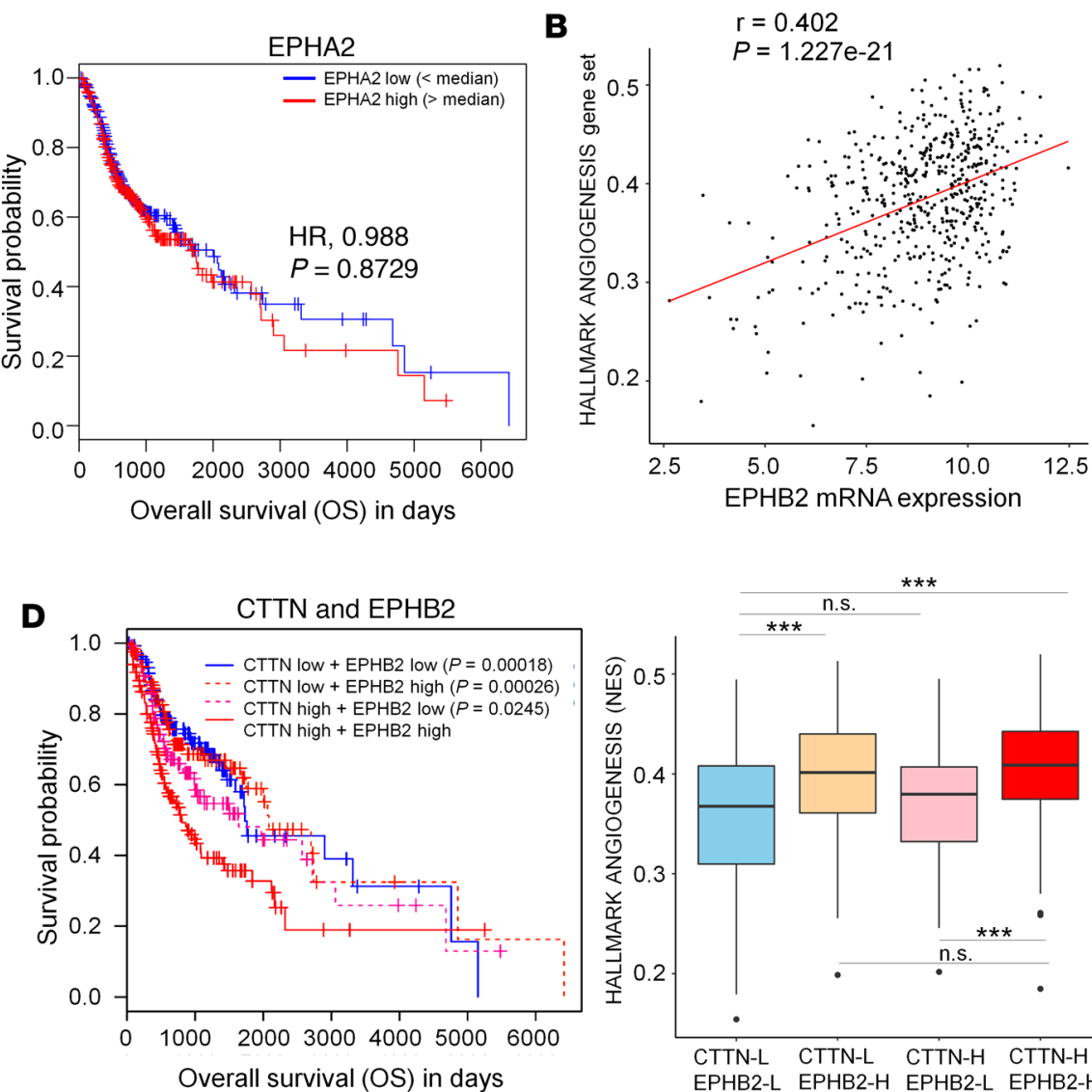

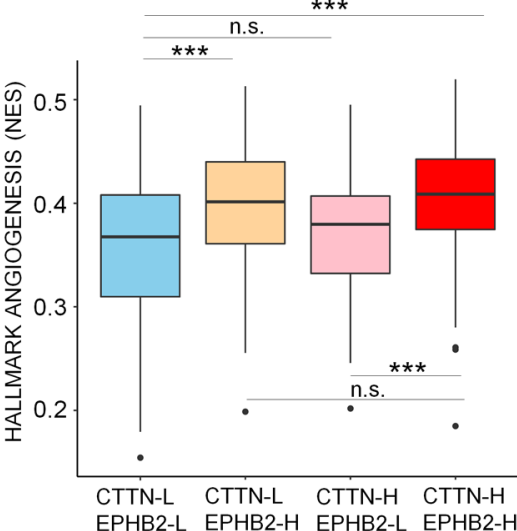

Results from 103 patients with oral cavity squamous cell carcinoma

$\mathbf{E}$

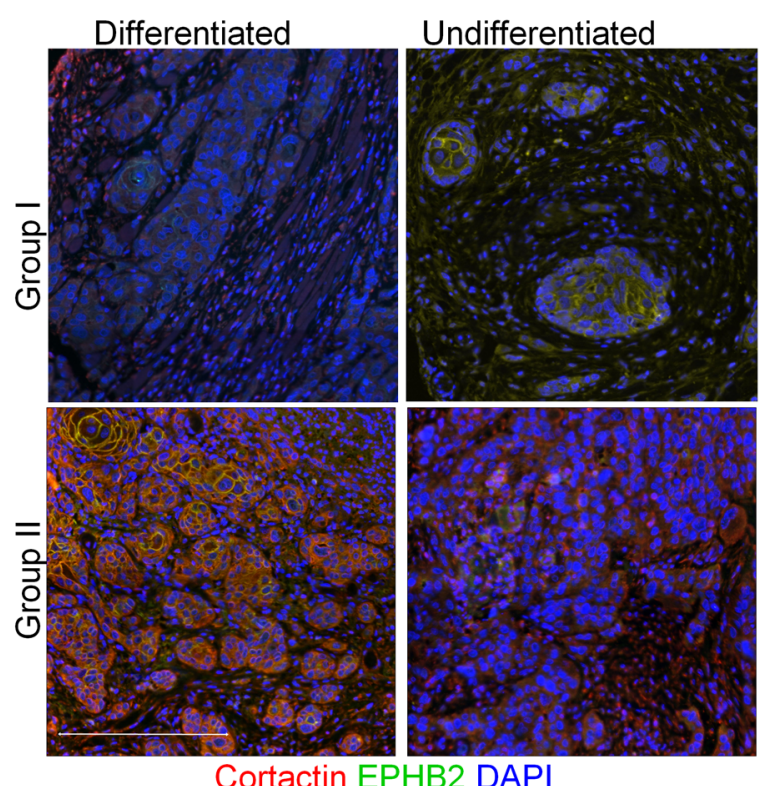

EPHB2

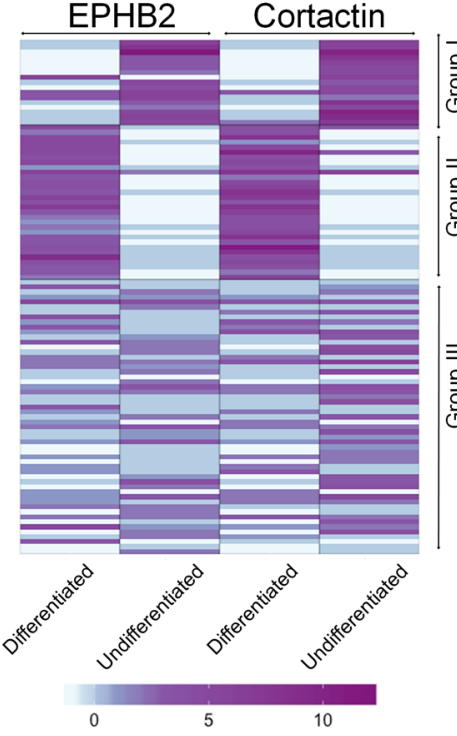

$\underline{\text { Regional Distant metastasis }}$

$9 / 19$

$10 / 19$

$7 / 32$

$6 / 32^{*}$

$5 / 52^{* * *} \quad 3 / 52^{* * *}$

Figure 2. EPHB2 overexpression in HNSCC tumors correlates with patient survival and tumor angiogenesis. (A-D) Analysis of TCGA RNA-Seq expression data, provisional HNSCC tumor data set. (A) Kaplan-Meyer analysis of relationship between EPHB2 or EPHA2 expression in HNSCC tumors to overall survival. The association of clinical parameters with survival was computed by univariate Cox regression; $n=528$. (B) Positive correlation between the hallmark angiogenesis gene set and EPHB2 mRNA expression in tumors. Pearson correlation $r=0.402, P=1.227 \times 10^{-21} ; n=528$. (C) Relationship between cortactin (CTTN) RNA expression in HNSCC tumors and overall survival. The association of clinical parameters with survival was computed by univariate Cox regression; $n=528$. (D) Left: Synergistic effect of EPHB2 and CTTN expression in HNSCC tumors on overall patient survival. The association of clinical parameters with survival was computed by univariate Cox regression; $n=528$. Right: Correlation of EPHB2 and CTTN mRNA expression with the hallmark angiogenesis gene set in HNSCC tumors. $Y$ axis is the normalized enrichment score (NES) of hallmark angiogenesis gene set in respective groups. Single-sample gene set enrichment analysis (ssGSEA) was performed for statistical analysis; $n=528$. (E) Multiplex staining for EPHB2 (green) and CTTN (red) 
in a TMA generated from surgical HNSCC specimens (103 patients). Representative staining for patient samples in clusters I and II is shown for differentiated (left panels) and undifferentiated (right panels) HNSCC areas; see also Supplemental Figure 3 for unmerged representative images. Scale bar: $100 \mu$ m. Cumulative data of intensity scoring (see Methods) from the TMA is displayed as a heatmap, with unsupervised clustering revealing 3 distinct clusters. The ratios in cluster I to the other clusters were compared separately using Fisher's test. The 2 -sided $P$ values are Regional I vs. II, $0.07 ;$ I vs. III, $0.001 ;$ II vs. III, 0.20; Distant I vs. II, 0.03; I vs. III, 0.0001; II vs. III, 0.08. ${ }^{* *} P<0.001$; significance shown in $\mathbf{E}$ is in comparison with cluster I.

miRNAs are not responsible for HNSCC SEV-mediated angiogenesis. A number of reports have implicated miRNAs as EV cargoes regulating angiogenesis $(17,18,63)$. In addition to inducing ephrin reverse signaling, EPHB2 on SEVs could potentially drive angiogenesis by enhancing binding and uptake of SEVs by target cells in order to deliver miRNAs. To determine whether miRNAs are part of the EV-driven angiogenesis we observed, we inhibited miRNA maturation in OSC19 cells by depletion of DICER with shRNA (Supplemental Figure 6A) and purified SEVs from those cells. To confirm the effect of DICER depletion on the levels of mature miRNAs in SEVs, we quantitated total RNA levels, as well as candidate mature and pre-miRNA levels, in SEVs purified from control and DICER-KD cells. For candidate miRNAs, we chose miR-155 and miR-21, based on their known expression in $\operatorname{HNSCC}(64,65)$ and their previous association with angiogenesis $(66,67)$. Consistent with the $>90 \% \mathrm{KD}$ of DICER in cells (Supplemental Figure 6A), mature miRNA was decreased and pre-miRNA was increased in EVs purified from DICER-KD cells (Supplemental Figure 6B). Analysis of the total tube length and numbers of junctions of HUVECs treated with EVs from DICER-KD cells did not show significant changes compared with that of HUVECs treated with control EVs (Supplemental Figure 6C). These data suggest that delivery of miRNA is unlikely to be the mechanism by which EPHB2 on HNSCC SEVs promotes angiogenesis.

EV-carried EPHB2 promotes angiogenesis by inducing ephrin reverse signaling. Our initial characterization of OSC19 SEVs showed that they can induce ephrin-B phosphorylation (Figure 3B), an early step in ephrin reverse signaling. To further determine whether EPHB2 carried on SEVs induces ephrin reverse signaling, HUVECs were incubated with scrambled control, EPHB2-KD, or EPHB2-OE SEVs purified from OSC19 cells for 30 minutes, followed by Western blot analysis for downstream signaling events. Compared with PBS, control SEVs stimulated ephrin-B phosphorylation (Figure 5A). Consistent with an important role of EPHB2, incubation of HUVECs with SEVs purified from EPHB2-OE and -KD cells, respectively, increased and decreased phospho-ephrin-B levels in HUVECs. Phosphorylation of STAT3, which is known to be stimulated by ephrin-B reverse signaling (68), was also increased by SEVs in an EPHB2-dependent manner (Figure 5A). Interestingly, phosphorylation of VEGFR2 was not increased by SEV treatment (Figure 5A). Since STAT3 phosphorylation was strongly induced by SEVs, dependent on their EPHB2 content, we tested whether inhibition of STAT3 affects SEV-induced tube formation. Indeed, inhibition with the small molecule inhibitor Stattic (69) greatly reduced both tube length and junction number (Figure 5B). These data indicate that EPHB2 carried on SEVs induces angiogenesis by activation of ephrin reverse signaling and downstream STAT3 activation.

\section{Discussion}

In this study, we found that a key Eph receptor is exported in HNSCC SEVs and drives tumor-associated angiogenesis. Thus, blocking EphB-ephrin-B interactions by preincubation of SEVs with Fc-ephrin-B2 or by $\mathrm{KD}$ of EPHB2 inhibited SEV-induced angiogenesis, both in vitro and in vivo. Furthermore, EPHB2 expression in HNSCC cells promotes tumor angiogenesis in an orthotopic model. Mechanistically, EPHB2-positive SEVs induce ephrin reverse signaling, as assessed by phosphorylation of ephrin-B and STAT3. Finally, inhibition of STAT3 activity greatly reduced SEV-induced angiogenesis. These data suggest a model in which EV-carried EPHB2 can induce signaling at a distance to recruit blood vessels into tumors, where they facilitate tumor survival and metastasis (Figure 5C).

Our model, in which key transmembrane or membrane-linked molecules are transported by EVs, may help to explain the unique role that EVs appear to play in driving angiogenesis. Thus, EV-transported membrane-linked molecules could synergize with soluble, diffusible secreted factors. For example, ephrin-B reverse signaling is known to synergize with VEGF signaling, but traditional models would limit Eph-ephrin signaling to cell-to-cell contacts. By contrast, EVs secreted from the center of a hypoxic tumor could reach the tumor edge and beyond to stimulate angiogenic sprouting toward the source of secreted factors. Gradients of EVs could provide stable cues that synergize with soluble angiogenic factors such as VEGFs or angiopoietins. 
A

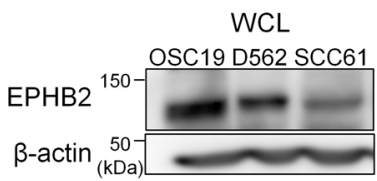

SEVS

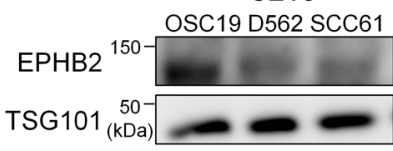

$\mathrm{TSG} 101_{(\mathrm{KDa})}^{50-} \longrightarrow$

TSG101 (kDa)
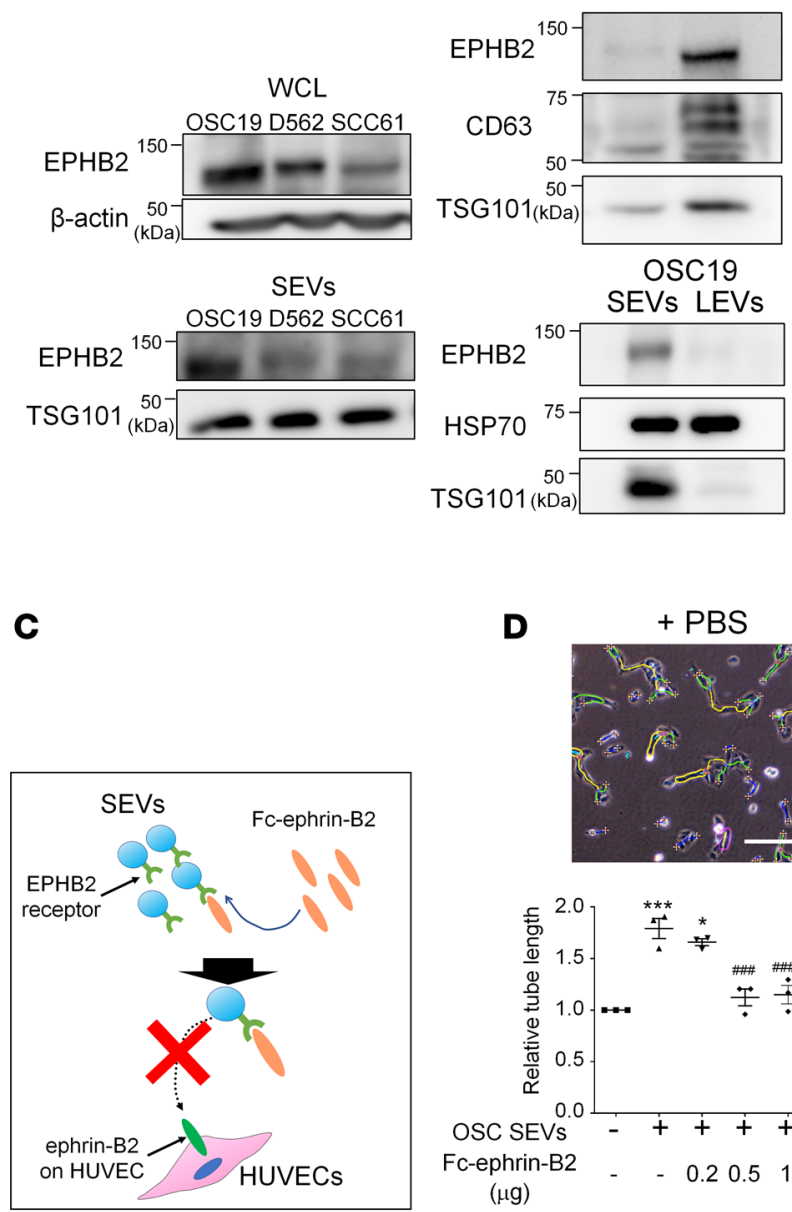

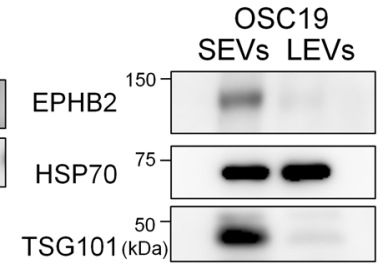

OSC19

WCL SEVs

OSC19

D

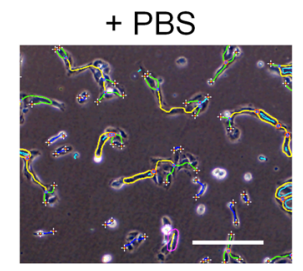

$$
\text { (2) }
$$

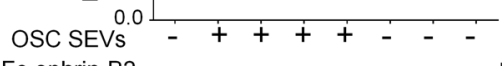
Fc-ephrin-B2

$(\mu \mathrm{g})$

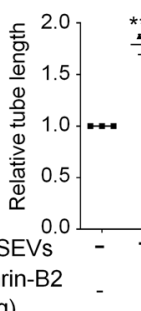

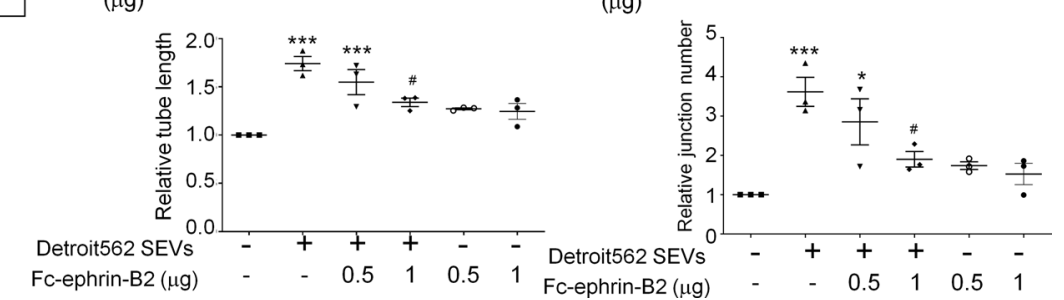

B
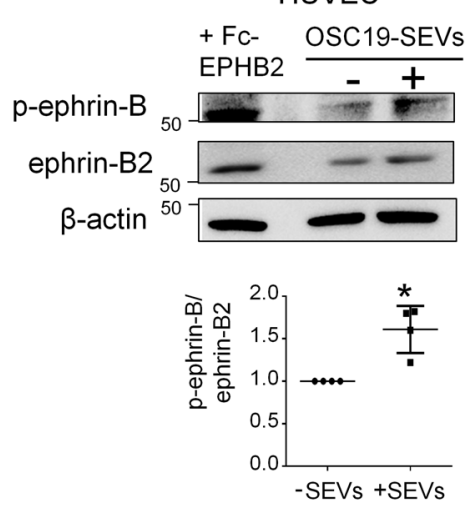

Figure 3. SEVs carry EPHB2 and induce ephrin reverse signaling. (A) Left: Representative Western blots of EPHB2 in whole cell lysates (WCL) and SEVs of OSC19, Detroit 562, and SCC61. Right top: Western blot comparing EPHB2, CD63, and TSC101 in 0SC19 whole cell lysates and SEVs (loading $5 \mu$ in each lane). Right bottom: Western blot comparing EPHB2, HSP70, and TSC101 in OSC19 SEVs and LEVs (loading $5 \mu$ in each lane). (B) Top: Representative Western blots of phospho-ephrin-B (p-ephrin-B), ephrin-B2, and $\beta$-actin in cell lysates from HUVECs treated with Fc-EPHB2, PBS (-), or $5 \times 10$ OSC19 SEVs $(+)$, as indicated. Bottom: p-ephrin-B/ephrin-B2 ratio from 3 independent experiments (median and 25th-75th percentile). Dunnett's method was used for statistical analysis. (C) Schematic of blocking experiments in which preincubation of Fc-ephrin-B2 with SEVs binds EPHB2 and blocks its binding to ephrin-B ligands on HUVECs. (D) Tube-formation assay. HUVECS were cultured with PBS (control), OSC19 SEVs with or without preincubation with recombinant ephrin-B2 (Fc-ephrin-B2), or Fc-ephrin-B2 alone. Top: Representative images. Scale bar: $500 \mu \mathrm{m}$. Bottom: Quantification of relative total tube length and junction number. Dot plots represent median and 25th-75th percentile. Tukey-Kramer method was used for statistical analysis using the average of $\geq 3$ technical replicates per condition for each $n$ value from $\geq 3$ independent experiments. ${ }^{*} P<0.05 ;{ }^{* *} P<0.001$ when compared with no added SEVs condition; $\# P<0.05 ; \# \# P<0.001$ when compared with + SEV s condition.

Previous studies have also shown that EVs derived from both cancer and normal cells (including mesenchymal stem cells, fibroblasts, microglia, and endothelial progenitor cells) play a key role in angiogenesis (70-73). Some previously reported angiogenic protein EV cargoes include IL-8, IGFBP1, IGFBP3, carbonic anhydrase 9, annexin II, myoferlin, WNT4, VEGFA, and VEGFD (13, 19, 20, 74-76). From our proteomics analysis of HNSCC EVs, IL-8, IGFBP1, carbonic anhydrase 9, WNT4, and VEGFR were not observed (Table 1). VEGFA, VEGFC, IGFBP3, annexin II, and myoferlin were observed in at least 1 
A

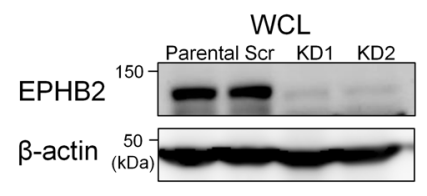

SEVS

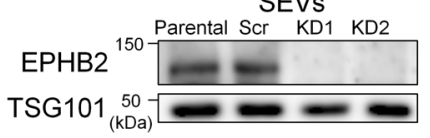

B
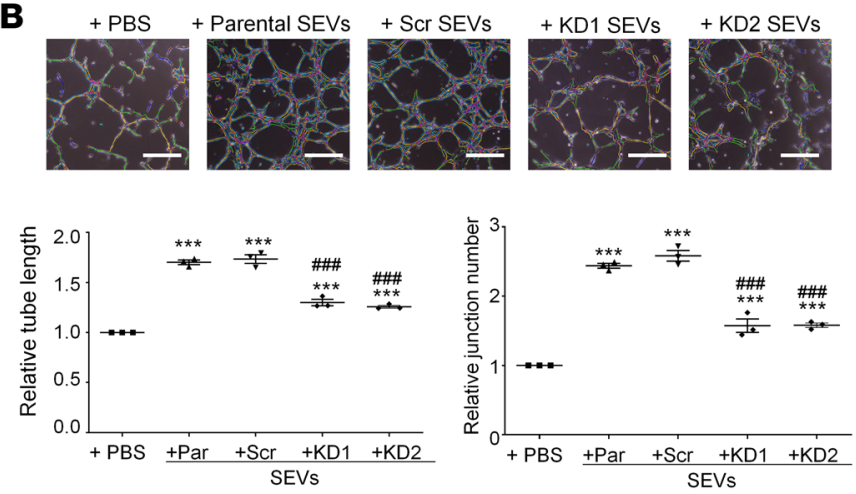
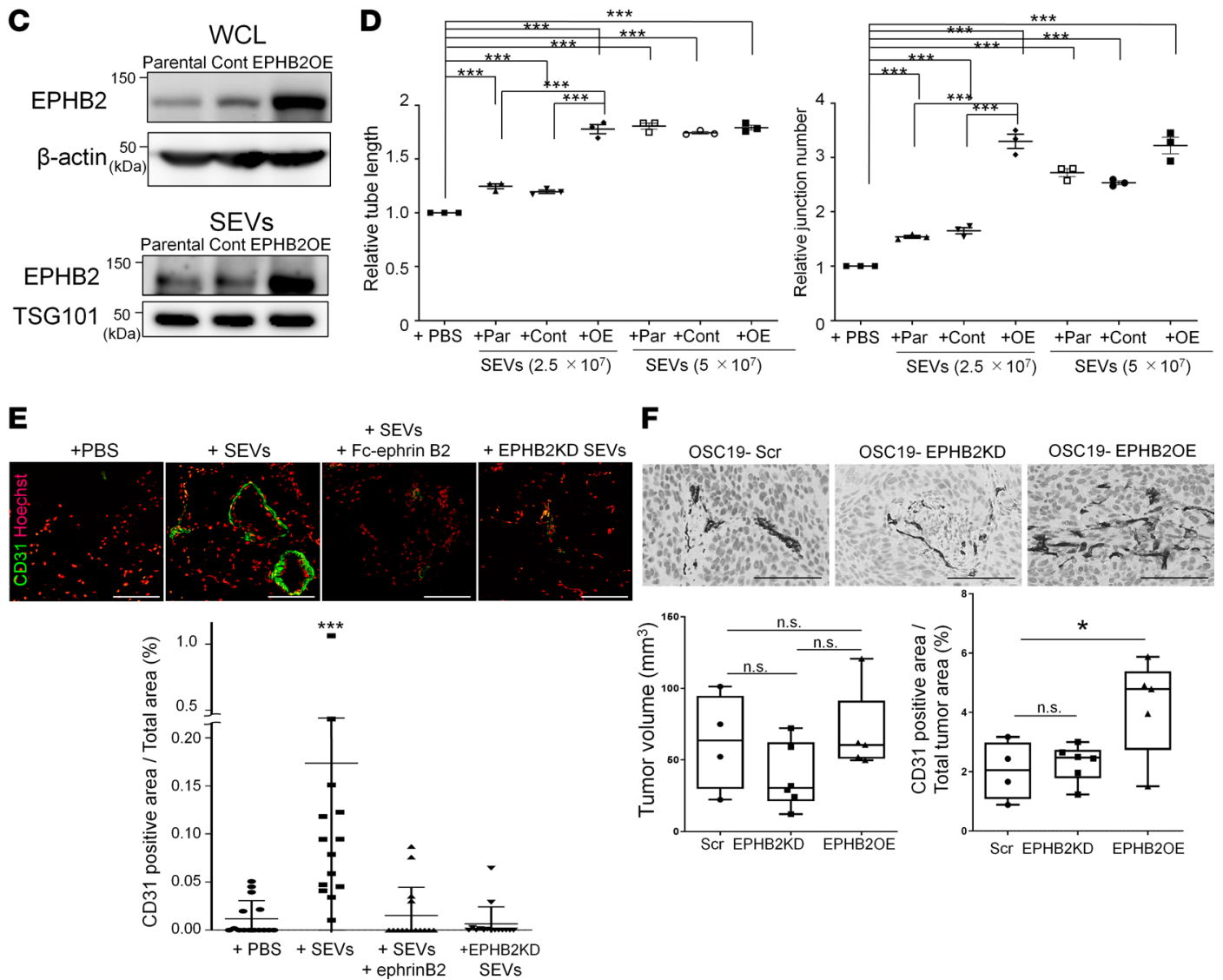

\section{$\mathbf{F}$}



Figure 4. EPHB2 on SEVs drives in vitro tube formation and in vivo angiogenesis. (A) Representative Western blot of EPHB2 in parental (control), scrambled control (SCR), and EPHB2-KD OSC19 whole cell lysates and SEVs. $\beta$-Actin and TSC101 serve as loading controls. (B) Tube-formation assay. Top: Representative images. Scale bar $=500 \mu \mathrm{m}$. Bottom: Quantification of relative total tube length and junction number. Plots show the average of $\geq 3$ technical replicates per condition for each $n$ value from $\geq 3$ independent experiments. ${ }^{* * *} P<0.001$ comparing PBS control with other groups. \#\#\# $P$ $<0.001$ comparing +Scr SEVs with +KD SEVs groups. (C) Western blot analysis of EPHB2, $\beta$-actin, and TSG101 in parental, empty vector control (Cont), and EPHB2-overexpression (EPHB2OE) OSC19 whole cell lysates and SEVs. (D) Analysis of tube-formation assay from HUVECs treated with the indicated numbers and types of SEVs. Plots show the average of $\geq 3$ technical replicates per condition for each $n$ value from $\geq 3$ independent experiments. (E) Matrigel plug assay in which SEVs or PBS were mixed with Matrigel and implanted s.c. in nude mice before harvesting and staining for blood vessels (CD31, green) or nuclei (Hoechst 33342, red). Top: Representative images of stained Matrigel plug tissue. Bottom: Quantification of CD31+ vessel area per total tumor area in Matrigel plugs. Dot plot shows mean \pm SEM. Dunnett's method was used for statistical analysis; $n \geq 15$ images per condition in 5 samples from 2 independent experiments. ${ }^{* *} P<0.001$ comparing PBS control with other groups. (F and G) Control (Scr), EPHB2-KD, and EPHB2-0E HNSCC cells were implanted in the tongues of nude mice. (F) Top: Representative images and quantitation of CD31 staining (black) in tongue tumors. Scale bar: $100 \mu \mathrm{m}$. Bottom: Quantitative analysis of tumor volume and CD31-positive area/tumor area in each group; $n=5 \mathrm{images}$ (1 image each from 5 tumors, whole tumor sections scanned for image quantitation). Frequency of lymph node metastasis showed $0 / 10$ for SCR, 0/10 for KD, and 1/10 for OE. For B, D, E, and F, box-and-whisker plots show median and 25th-75th percentile. Dunnett's method was used in $\mathbf{B}$ and $\mathbf{E}$, and Tukey-Kramer method was used in $\mathbf{D}$ and $\mathbf{F}$ for statistical analysis. ${ }^{*} P<0.05{ }^{* *} P<0.001$. 
A
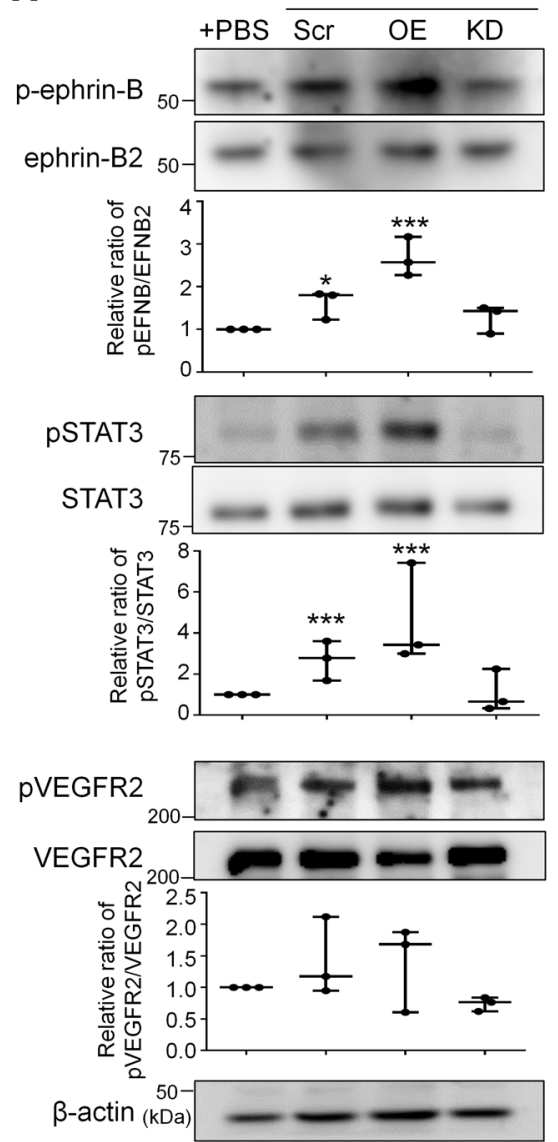

C



+ Stattic
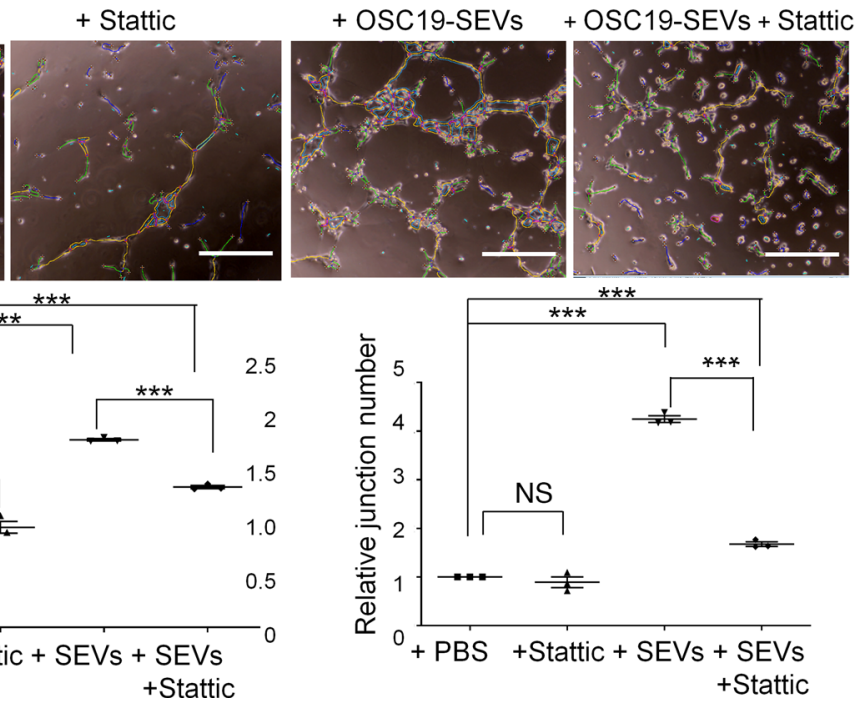

Figure 5. EPHB2 on SEVs drives an ephrin-B-STAT3 angiogenic signaling cascade. (A) Representative Western blots of phosphorylated ( $p$-ephrin-B, p-STAT3, p-VEGFR2) and total (ephrin-B2, STAT3, VEGFR2, $\beta$-actin) proteins in cell lysates from HUVECs treated with PBS or SEVs, as indicated. Graphs show normalized quantitative analyses of the ratio of phosphorylated to total proteins from $n=3$ Western blots. Box-and-whisker plots show median and 25th-75th percentile. Dunnett's method was used for statistical analysis. ${ }^{*} P<0.05,{ }^{* * *} P<0.001$. (B) Tube-formation assay of HUVECs treated with SEVs in the presence or absence of the Stat3 inhibitor Stattic. Representative images and analysis. Scale bar: $500 \mu \mathrm{m}$. Box-and-whisker plots show median and 25th-75th percentile. Tukey-Kramer method was used for statistical analysis using the average of $\geq 3$ technical replicates per condition for each $n$ value from $\geq 3$ independent experiments. ${ }^{* *} P<0.001$. (C) Model of SEV-induced angiogenesis pathway. EPHB2 on cancer cellderived SEVs binds to ephrin-B2 on endothelial cells and activates ephrin-B2 reverse signaling with downstream phosphorylation and activation of STAT3. While phosphorylation of VEGFR2 downstream of ephrin-B2 reverse signaling has been reported (55), we did not detect changes due to SEV treatment, suggesting selective activation of ephrin reverse signaling pathways by SEVs. 
HNSCC EV sample, and VEGFA and IGFBP3 have been previously reported to promote angiogenesis in HNSCC $(77,78)$. In this manuscript, we focused on receptor-ligand transmembrane molecules that could not be transported in any other way than via EVs and that could specifically target the EVs to endothelial cells. Furthermore, in separate antibody array experiments, we did not observe significant amounts of VEGFs or IGFBP3 associated with HNSCC SEVs (not shown), suggesting that only minimal amounts associate with SEVs or that they are lost during our purification process. However, it is certainly possible that additional EV cargoes may promote angiogenesis in HNSCC tumors, and some cytoplasmic cargoes could even be carried in the same vesicles as ephrin receptors and ligands.

In addition to protein cargoes, a number of EV-derived miRNAs have been shown to promote angiogenesis $(9,79)$. Using DICER-KD to prevent maturation of pre-miRNAs in HNSCC cells, we detected no effect of $\mathrm{KD}$ EVs on endothelial tube formation. While our result does not rule out EV-delivered miRNAs being important angiogenic regulators in other systems, it does suggest that it is not a major driver in our system.

Eph receptors are a subfamily of receptor protein-tyrosine kinases (80) and were first identified as regulators of cell-to-cell interactions in the nervous system $(81,82)$, including axon guidance and branching, and development of neuronal growth cones. More recently, $\mathrm{KO}$ mouse studies identified EphB-ephrin-B signaling as a key regulatory control for development of blood and lymph vessels, due to interactions between EPHB4 and ephrin-B2 on venous and arterial endothelial cells, respectively $(32,55)$. In one of those studies, ephrin-B reverse signaling was also shown to be important for tumor angiogenesis (55). Additional EphB receptors, including EPHB2, may act in a similar manner in remodeling the embryonic vascular system (34). Ephrins are also involved in cell migration, cell fate, and immunity $(83,84)$.

Our study is the first to our knowledge to identify a role for EphB-ephrin-B interactions in HNSCC-induced tumor angiogenesis. Furthermore, our study is the first to our knowledge to identify ephrin family molecules on EVs as key angiogenesis regulators. Consistent with our finding that SEVs can induce ephrin reverse signaling in endothelial cells, a recent study showed that exosomal EPHB2-ephrin-B1 reverse signaling can induce neuronal repulsion (85). These results suggest that EV-based ephrin signaling may be a general paradigm that could mediate ephrin signaling in a variety of contexts, including axon guidance, angiogenesis, and potentially immune cell interactions (86-89).

Consistent with our TCGA analysis (Figure 2A), EPHB2 overexpression in several other cancer types has been reported to correlate with decreased patient survival $(90,91)$. We further found that the correlation of EPHB2 overexpression in HNSCC tumors with poor prognosis was even higher if the exosome secretion regulator cortactin (31) was also overexpressed, suggesting that exosomal EPHB2 may drive tumor aggressiveness. Our findings suggest that induction of tumor angiogenesis could be an important component of that process. Future studies should further elucidate the role of EV-carried EPHB2 and other EV-associated ephrin family molecules in driving near- and long-distance signaling in cancer.

Anti-angiogenesis therapy has, thus far, been employed in a subset of cancers and largely consists of anti-VEGF antibody or kinase inhibitor therapy against VEGFR signaling (22, 92-98). In HNSCC, a number of trials testing anti-angiogenesis therapies are either completed or ongoing; however, the effects have been limited (99-104). In the most recent phase III trial, conventional therapy with the monoclonal anti-VEGF antibody bevacizumab did not show significant improvement in overall or disease-free survival compared with conventional therapy alone (105). In fact, in the latest national comprehensive cancer network guidelines for HNSCC therapy, no anti-VEGF reagents were recommended (106), despite strong association of numerous angiogenesis regulators with poor prognosis in $\operatorname{HNSCC}(107,108)$. These data suggest that new targets are needed. One possibility is to test whether EV-based targets such as EPHB receptors or ligands that should synergize with VEGF would lead to greater efficacy.

In summary, we find that EPHB2 carried on HNSCC-secreted EVs can induce ephrin-B reverse signaling and tumor angiogenesis. This mechanism may be important for understanding the HNSCC microenvironment and may also be a potential target for future cancer therapy.

\section{Methods}

Supplemental Methods are available online with this article.

Antibodies. phosphorylated ephrin-B (3481, rabbit polyclonal), EPHB2 (D2X2I, 83029, rabbit monoclonal), phosphorylated STAT3 (Tyr705, D3A7, 9145, rabbit monoclonal), STAT3 (124H6, 9139, mouse monoclonal), phosphorylated VEGFR2 (Ty1175, 19A10, 2478, rabbit monoclonal), and VEGFR2 (55B11, 2479, rabbit monoclonal) were purchased from Cell Signaling Technology. Ephrin-B2 
(EPR10072[B], ab150411, mouse monoclonal), CD31 (EPR3094, ab76533, rabbit monoclonal), and cortactin (4F11, ab33333, mouse monoclonal) were purchased from Abcam. EPHB2 (AF467, goat polyclonal) and goat IgG HRP conjugated antibody (HAF017, rabbit polyclonal) were purchased from R \& D systems. EPHB4 (3D7G8, 37-1800, mouse monoclonal), Alexa Fluor 750 goat anti-rabbit secondary antibody (A21039), Alexa Fluor 647 goat anti-mouse secondary antibody (A32728), and Alexa Fluor 488 goat anti-rabbit secondary antibody (A27034) were purchased from Thermo Fisher Scientific. $\beta$-Actin (AC-74, A5316, mouse monoclonal) was purchased from Sigma-Aldrich. Anti-rabbit IgG (H+L) HRP conjugate (W4011) and anti-mouse IgG $(\mathrm{H}+\mathrm{L}) \mathrm{HRP}$ conjugate (W4021) were purchased from Promega. Hemoglobin $\beta / \gamma / \delta / \varepsilon$ antibody (A-8) Alexa Fluor (A-8, sc-390668, mouse monoclonal) was purchased from Santa Cruz Biotechnology Inc. For Western blot, antibodies were diluted 1:2000-1:10,000. For IHC or immunofluorescent staining, antibodies were diluted 1:200-1:1000.

Cell lines and constructs. HUVECs (Lonza) were maintained in EBM-2 complete endothelial growth medium (Cambrex Corporation) containing 2\% FBS, human recombinant VEGF (rhVEGF), basic FGF (bFGF), hEGF, insulin-like growth factor 1 (IGF-I), hydrocortisone, ascorbic acid, heparin, and GA-1000 (gentamicin and amphotericin B, $1 \mu \mathrm{g} / \mathrm{mL}$ ) according to supplier instructions. HMVECs-dLyAd (Lonza) were maintained in EBM-2 Basal Medium (Lonza), 5\% FBS, hydrocortisone, hFGF, VEGF, IGF-1, ascorbic Acid, hEGF, and GA-1000 according to supplier instructions. HUVECs and HMVECs-dLyAd were used at passage 6 or less. OSC19, Detroit 562, and SCC61 cells were a gift from Wendell Yarbrough (University of North Carolina at Chapel Hill School of Medicine, Chapel Hill, North Carolina, USA) and were maintained in DMEM with 20\% FBS with $0.4 \mu \mathrm{g} / \mathrm{mL}$ hydrocortisone. MOC1 and MOC2 cells were obtained from Young Kim (Vanderbilt University Medical Center, Nashville, Tennessee, USA) and were cultured in IMDM/F12 at a 2:1 mixture with 10\% FBS, $5 \mathrm{ng} / \mathrm{mL}$ EGF, $400 \mathrm{ng} / \mathrm{mL}$ hydrocortisone, and $5 \mathrm{mg} / \mathrm{mL}$ insulin (Sigma-Aldrich). Cells were cultured on $100 \mathrm{~mm}$ tissue culture plates and grown under $5 \% \mathrm{CO}_{2}$ at $37^{\circ} \mathrm{C}$. Medium was renewed every 3 days, and cells were split every 4 days. Stable $\mathrm{KD}$ or stable expression of genes was achieved using the ViraPower Lentiviral expression system (Thermo Fisher Scientific) according to the manufacturer's protocol. Gene manipulation from polyclonal stable cell lines was checked by Western blot, and cells were passaged for no longer than a month before discarding and thawing early-passage cultures. Cells were periodically checked for mycoplasma contamination. Lentiviral shRNA constructs for DICER (KD1: TRCN0000051260, 5' - CCGGCCACACATCTTCAAGACTTAACTCGAGTTAAGTCTTGAAGATGTGTGGTTTTTG - 3'; and KD2: TRCN0000051262, 5' - CCGGGCTGGCTGTAAAGTACGACTACTCGAGTAGTCGTACTTTACAGCCAGCTTTTTG - 3'), and EPHB2 (KD1: TRCN0000006423， 5' - CCGGGCTAGACAAGATGATCCGCAACTCGAGTTGCGGATCATCTTGTCTAGCTTTTT - 3'; and KD2: TRCN0000006426, 5' - CCGGCGGGAGTTTGCCAAGGAAATTCTCGAGAATTTCCTTGGCAAACTCCCGTTTTT - 3') were from MilliporeSigma. The stable EPHB2 overexpression construct was made by cloning the open reading frame of EPHB2 downstream of the CMV promoter into pLOC lentiviral vector (Dharmacon).

Isolation of EVs from conditioned medium and EV characterization. Cells were cultured to $80 \%$ confluence and washed with PBS before culturing for 48 hours in Opti-MEM, which is a serum-free but growth factor-containing medium. Conditioned medium was subjected to serial centrifugation to respectively sediment live cells (300 $g$ for 5 minutes), dead cells (2,500 $g$ for 25 minutes), and LEVs (10,000 $g$ for 30 minutes in Ti 45 rotor; Beckman Coulter). To obtain SEVs, we used a cushion-density gradient protocol (29). Briefly, $30 \mathrm{~mL}$ of the supernatant from the $10,000 \mathrm{~g}$ centrifugation step was overlaid onto $2 \mathrm{~mL}$ of OptiPrep ( $60 \% \mathrm{wt} / \mathrm{vol}$ aqueous iodixanol; Axis-Shield PoC) and centrifuged at 100,000 $\mathrm{g}$ for 4 hours in an SW32 rotor (Beckman Coulter). After the spin, $1 \mathrm{~mL}$ medium from the bottom of the tube was mixed with $2 \mathrm{~mL}$ OptiPrep to make a SEV-Optiprep mixture with a final $40 \% \mathrm{wt} / \mathrm{vol}$ concentration of iodixanol. This mixture was then subjected to density gradient ultracentrifugation by adding it into the bottom of a $14 \times 89-\mathrm{mm}$ tube and overlaying OptiPrep solutions (20\% wt/vol, $10 \% \mathrm{wt} / \mathrm{vol}$, and $5 \% \mathrm{wt} / \mathrm{vol})$ diluted with $0.25 \mathrm{M}$ sucrose $/ 10 \mathrm{mM}$ Tris, $\mathrm{pH}$ 7.5. A continuous gradient from 40\%-5\% was made through centrifugation at 100,000 $g$ (24,000 rpm in SW40 rotor) for 18 hours. Twelve density gradient fractions were collected and diluted in PBS before pelleting through another round of centrifugation at 100,000 $\mathrm{g}$ for 3 hours, followed by washing and resuspension in PBS.

Analysis of size and protein concentration of EVs. All EV preparations were analyzed for size and concentration by nanoparticle tracking with a Zetaview analyzer (Particle Metrix $\mathrm{GmbH}$ ) as recommended by the company. EV protein levels were determined using the microBCA Protein Assay Reagent kit (Pierce) according to the manufacturer's instructions. 
Transmission electron microscopy analysis. Transmission electron microscopy (FEI/Philips CM200 FEG) was performed on purified EVs. For negative staining of purified EVs, Formvar carbon film-coated grids (FCF-200-Cu; Electron Microscopy Sciences) were washed in Milli-Q water (MilliporeSigma), followed by $100 \%$ ethanol. For each step, excess liquid was removed by wicking with filter paper. Samples $(10-\mu \mathrm{L})$ were added to grids for 30 minutes. Grids were immediately stained with $2 \%$ phosphotungstic acid, $\mathrm{pH} 6.1$, for 20 seconds and allowed to dry overnight. Grids were imaged using a FEI Tecnai T12 transmission electron microscopy (120 kV LaB6 source; Thermo Fisher Scientific), Gatan cryo-transfer stage (Thermo Fisher Scientific), and AMT XR41-S side-mounted 2K × 2K CCD camera, 2102 SC (Advanced Microscopy Techniques). All SEV and LEV images were collected at $\times 110,000$ with the CCD camera.

Western blot analysis. Cells were lysed in $50 \mathrm{mmol} / \mathrm{L}$ Tris- $\mathrm{HCl}$ ( $\mathrm{pH} 7.4), 1 \% \mathrm{NP} 40,0.25 \%$ sodium deoxycholate, $150 \mathrm{mmol} / \mathrm{L} \mathrm{NaCl}, 1 \mathrm{mmol} / \mathrm{L}$ EDTA, $1 \mathrm{mmol} / \mathrm{L}$ phenylmethylsulfonyl fluoride (PMSF), $1 \mu \mathrm{g} /$ $\mathrm{mL}$ pepstatin, $1 \mathrm{mmol} / \mathrm{L}$ sodium orthovanadate (Sigma-Aldrich). EVs were lysed in $1 \% \mathrm{SDS}$. For Western blot analysis, $40 \mu \mathrm{g}$ (for cell lines) or $5 \mu \mathrm{g}$ (for EVs) protein was resolved on $6 \%$ or $10 \%$ SDS-polyacrylamide gels and transferred to nitrocellulose membranes. Western blot signals were detected with Pierce Western Blot Signal Enhancer (Thermo Fisher Scientific) and imaged on a chemiluminescence imager (Amersham imager 600, GE Healthcare Life Sciences)

Endothelial tube-formation assay. HUVECs or HMVECs-dLyAd $\left(5 \times 10^{4}\right.$ per well) were suspended in $400 \mu \mathrm{L}$ of Opti-MEM medium containing 10\% EBM-2 medium without serum and were gently added to Matrigel-coated wells in a 24-well plate. Cells were treated with $5 \times 10^{7}$ per well of tumor-derived SEVs or LEVs in $5 \mu \mathrm{L}$ of PBS or PBS alone. For the experiments using Fc-ephrin-B2, HUVECs were cocultured with $5 \times 10^{7}$ purified HNSCC EVs with or without preconjugation (for 10 minutes) of various concentrations of recombinant human Fc-ephrin-B2 chimera protein ( $\mathrm{R} \& \mathrm{D}$ Systems). As a positive control for ephrin-B phosphorylation, HUVECs were cocultured with $15 \mu \mathrm{g}$ recombinant human Fc-EPHB2 chimera protein (R \& D Systems). For inhibition of STAT3 activity, cells were treated with $200 \mathrm{nM}$ Stattic (2798, Tocris). After 12 hours, images of cells were captured using phase contrast microscopy $(\times 10 / 0.25$ N.A. Plan PH2 objective lens, EVOS XL Core Imaging System; Thermo Fisher Scientific). Total tube length and total junction number were calculated automatically using the angiogenesis analyzer plugin in ImageJ (NIH; http://image.bio.methods.free.fr/).

Animals. Seven-week-old female athymic nude mice and C57BL/6 mice were purchased from Charles River Laboratory and kept in a pathogen-free facility approved by the American Association for the Accreditation of Laboratory Animal Care that met all current regulations and standards of the U.S. Department of Agriculture, U.S. Department of Health and Human Services, and the National Institutes of Health. Mice were fed irradiated standard mouse chow (LabDiet) and autoclaved, reverse osmosis treated water.

Orthotopic nude mouse model of HNSCC. Subconfluent cultures by trypsinization and washed with PBS. Oral tongue tumors were established by injecting cells $\left(1 \times 10^{5}\right.$ for OSC19, Detroit 562, SCC61, and MOC1; $5 \times 10^{4}$ for MOC2) suspended in $30 \mu \mathrm{L}$ of serum-free DMEM into the tongues of nude (OSC19, Detroit 562, SCC61) or C57BL/6 (MOC1, MOC2) mice as described previously (109, 110). Mice were examined twice a week for tumor size and weight loss. Tongue tumor size was measured with microcalipers. Tumor volume was calculated as $(A)\left(B^{2}\right) \pi / 6$, where $A$ is the longest dimension of the tumor and $B$ is the dimension of the tumor perpendicular to A. Mice were sacrificed after 4 weeks (SCC61, OSC19, and Detroit 562) or 2 weeks (MOC1 and MOC2), and tongues were fixed, sectioned, and stained. Imaging of fluorescent CD31 staining, hemoglobin staining, and H\&E staining in tumors was performed using an Aperio Versa 200 scanner (Leica) in the Vanderbilt Digital Histology Shared Resource. Lymph node metastasis in H\&Estained cervical lymph nodes tissue was assessed using light microscopy.

Assessment of blood vessel density from tumor tissue. After scanning CD31-stained tumor tissue specimens, whole tumor area and CD31-stained area in the tumors was calculated using ImageJ. CD31-stained area/ whole tumor area $\times 100$ was assessed as blood vessel density.

Matrigel plug assay. The Matrigel plug assay was performed essentially as previously described (61). Briefly, growth factor-reduced Matrigel $(350 \mu \mathrm{L})$ was mixed in liquid form at $4^{\circ} \mathrm{C}$ with $50 \mu \mathrm{L}$ of EVs $(30$ $\mu \mathrm{g})$. The Matrigel-SEV mixture was injected s.c. into the flanks of 6- to 8-week-old athymic nude mice. After 21 days, mice were sacrificed, and the Matrigel plugs were excised and fixed in $4 \%$ formaldehyde in phosphate buffer. Plugs were embedded in paraffin, sectioned, and immunostained for CD31. Images were acquired with an A1R Confocal laser Microscope system (Nikon) equipped with a × 20/1.40 NA Plan Apo objective lens (Nikon), and analyzed using ImageJ. 
Tumor tissue microarray. After appropriate IRB approval for retrospective study of patient specimens and records, a tissue microarray (TMA) of 120 primary oral cavity SCCs was created from previously untreated patients with no history of cancer. After creation of the array, patient identifiers were purged so the specimens were deidentified. The array was assembled retrospectively from 1997-2011 from patients from databases from the Departments of Radiation Oncology, Otolaryngology, and Pathology at Washington University (Seattle, Washington, USA). For each patient, two (or four) 2-millimeter punches of representative blocks from their primary tumors were taken and placed on the array, depending on the amount of primary tumor tissue available. Diagnoses were confirmed by a reviewing pathologist. Pathology data was garnered from review of the pathology database original reports, clinical follow up from departmental databases, and review of the electronic medical records, including date of death where applicable. Multiplex immunofluorescent staining of a $6-\mu \mathrm{m}$ section of the tissue microarray was performed with antibodies for cortactin and EPHB2 in conjunction with collagen (CNA35) (111) and a nuclear dye (DAPI). Images were acquired by whole-slide scanning. Histological staining in differentiated and undifferentiated regions of the tumor tissue was scored for the percent of cells positive (score of $0-3$ ) and the intensity of fluorescent staining (score of 1-4) with the final score calculated as a multiplier of these 2 scores (score of 0-12). Quantitation for each protein was determined for differentiated and undifferentiated portions of the tissue from each patient. Tumor tissue staining scores were subsequently subjected to unsupervised clustering in R () to identify possible groups of patients with distinct EphB2 and cortactin staining patterns that might have corresponding changes in tumor dissemination.

Mass spectrometry and liquid chromatography tandem mass spectrometry analysis. Shotgun proteomic analysis of purified EVs was performed by first partially resolving the proteins by about $1.5 \mathrm{~cm}$ in a $10 \%$ Novex precast gel, excising the protein region, and then performing in-gel tryptic digestion to recover peptides. The peptides were analyzed via Multidimensional Protein Identification Technology essentially as described earlier $(112,113)$. Briefly, digested peptides were loaded onto a biphasic precolumn consisting of $4 \mathrm{~cm}$ of reversed phase material followed by $4 \mathrm{~cm}$ of strong cation exchange reversed phase material. Once loaded, this column was placed in line with a $20-\mathrm{cm}$ reversed phase analytical column packed into a nanospray emitter tip directly coupled to a linear ion trap mass spectrometer. A subset of peptides was eluted from the strong cation exchange (SCX) material onto the reversed phase analytical via a pulse of volatile salt. Those peptides were separated by a reversed phase gradient and then ionized directly into the mass spectrometry (MS). This proceeded for a total of 11 salt elution steps over the course of approximately 22 hours of data acquisition. Both the intact masses (MS) and fragmentation patters (MS/MS) of the peptides were collected in a data-dependent manner utilizing dynamic exclusion to maximize depth of proteome coverage. Resulting peptide MS/MS spectral data were searched against the human protein database, to which common contaminants had been appended using Sequest (114), and identifications were collated and filtered using Scaffold (www.proteomesoftware.com).

Statistics. Statistical analysis of overall survival and gene expression was assessed for The Cancer Genome Atlas (TCGA) patient data using Linkedomics (115) (http://linkedomics.zhang-lab.org/admin. php). Single-sample GSEA (ssGSEA) was performed on RNA-Seq data of HNSCC patients from TCGA database using from MSigDB hallmark angiogenesis gene set (116). A list of the gene set was shown in Supplemental Table 3. We calculated normalized angiogenesis enrichment score across the patients and compared it with patients categorized into cortactin-high (CTTN-high) and -low (greater than average CTTN mRNA expression, CTTN-H; lower than average mRNA expression, CTTN-L) and EPHB2 -high and -low (greater than average EPHB2 mRNA expression, EPHB2-H; lower than average mRNA expression, EPHB2-L) concurrently. Kaplan-Meier survival curves were evaluated using log-rank tests using gene expression dichotomized at the median. The association of clinical parameters with survival was computed by univariate Cox regression. The synergistic effect of 2 genes were analyzed by comparing median expression-based dichotomized patient groups. Hazard ratios were assessed by Cox regression method in $\mathrm{R}$ (https://www.R-project.org) using package survival (v2.44).

For experimental data analysis, GraphPad Prism 8 software (GraphPad Software) was used. For all tube-formation assays, the statistical analysis was based on the biological replicate average values, not on the technical replicate values. Normality assumption was tested using a 1-way ANOVA for all tube-formation assays, analysis of CD31 staining area, EV secretion rates, qPCR of miRNA results, tumor volumes, and Western blot results. Tukey-Kramer method was used for multiple comparison in the analysis of CD31 staining area, EV secretion rates, and tube-formation assays (Figure 1, C and D, and Figure 4, B and D). 
Dunnett's test was used to compare each of a number of treatments with a single control in the analysis of tube-formation assays, CD31 staining areas, and Western blot results (Figure 1F; Figure 3D; Figure 4, $\mathrm{E}$ and $\mathrm{F}$; and Figure 5A). $P$ value was evaluated by 2-tailed $t$ test in Figure 2, A, C, and D. $P<0.05$ was considered significant for all statistical analysis.

Study approval. Animal procedures were carried out according to a protocol approved by The Vanderbilt University's IACUC (approved protocol no. M1800027-00). For the TMA array analysis, The Washington University's IRB approved for retrospective study of patient specimens and records (approved IRB no. 201109193). Animal treatment was approved by the American Association for the Accreditation of Laboratory Animal Care and met all current regulations and standards of the US Department of Agriculture, US Department of Health and Human Services, and the NIH.

\section{Author contributions}

SS and AMW designed the experiments. SS conducted the majority of experiments. AE and AZ stained and analyzed the TMA sections. SV and BZ carried out bioinformatic and statistical analysis. YK and JSL supplied clinical materials and information. SS and AMW wrote the manuscript with input and editing from all coauthors.

\section{Acknowledgments}

We thank H. Takahashi, N. Tanaka, and J. Myers for help with the establishment of orthotopic model; P. Mulcrone for help with the establishment of tube-formation assay; S. Sinha and D. Hoshino for help with the method of EV isolation; and R. Ihrie for EVOS XL Core Imaging System help. Research was supported by the NIH under award numbers R01CA163592 and R01CA206458 to AMW, by Vanderbilt TIPS pilot funding to AZ and AMW (Single Cell Biology Project), and by a pilot voucher to SS from the Vanderbilt CTSA grant UL1 RR024975. YK was supported by the NIH under award numbers 1R01CA178613 and R01DE027749, as well as by Barry and Amy Baker Endowed Lab and Incyte IRG. SV and BZ were supported by the National Cancer Institute (NCI) Clinical Proteomic Tumor Analysis Consortium (CPTAC) grant U24CA210954 and the Cancer Prevention and Research Institutes of Texas (CPRIT) grant RR160027.

Address correspondence to: Alissa M. Weaver, 748 Preston Research Building, Vanderbilt University School of Medicine, Nashville, Tennessee 37212, USA. Phone: 615.936.3529; Email: alissa.weaver@vanderbilt.edu.

SV's current address is: Department of Translational Molecular Pathology, The University of Texas MD Anderson Cancer Center, Houston, Texas, USA.

1. McDermott JD, Bowles DW. Epidemiology of Head and Neck Squamous Cell Carcinomas: Impact on Staging and Prevention Strategies. Curr Treat Options Oncol. 2019;20(5):43.

2. Gillison ML, et al. Distinct risk factor profiles for human papillomavirus type 16-positive and human papillomavirus type 16-negative head and neck cancers. J Natl Cancer Inst. 2008;100(6):407-420.

3. Rettig EM, D'Souza G. Epidemiology of head and neck cancer. Surg Oncol Clin N Am. 2015;24(3):379-396.

4. Takes RP, et al. Distant metastases from head and neck squamous cell carcinoma. Part I. Basic aspects. Oral Oncol. 2012;48(9):775-779.

5. Sinha P, et al. Distant metastasis in p16-positive oropharyngeal squamous cell carcinoma: a critical analysis of patterns and outcomes. Oral Oncol. 2014;50(1):45-51.

6. Bauer E, et al. Extranodal extension is a strong prognosticator in HPV-positive oropharyngeal squamous cell carcinoma [Published online ahead of print May 11, 2019]. Laryngoscope. doi: 10.1002/1ary.28059.

7. Ding D, et al. Association Between Lymph Node Ratio and Recurrence and Survival Outcomes in Patients With Oral Cavity Cancer. JAMA Otolaryngol Head Neck Surg. 2019;145(1):53-61.

8. Maas SLN, Breakefield XO, Weaver AM. Extracellular Vesicles: Unique Intercellular Delivery Vehicles. Trends Cell Biol. 2017;27(3):172-188.

9. Sato S, Weaver AM. Extracellular vesicles: important collaborators in cancer progression. Essays Biochem. 2018;62(2):149-163.

10. Maji S, et al. Exosomal Annexin II Promotes Angiogenesis and Breast Cancer Metastasis. Mol Cancer Res. 2017;15(1):93-105.

11. Hsu YL, et al. Hypoxic lung cancer-secreted exosomal miR-23a increased angiogenesis and vascular permeability by targeting prolyl hydroxylase and tight junction protein ZO-1. Oncogene. 2017;36(34):4929-4942.

12. Chiba M, Kubota S, Sato K, Monzen S. Exosomes released from pancreatic cancer cells enhance angiogenic activities via dynamin-dependent endocytosis in endothelial cells in vitro. Sci Rep. 2018;8(1):11972.

13. Kucharzewska $P$, et al. Exosomes reflect the hypoxic status of glioma cells and mediate hypoxia-dependent activation of vascular cells during tumor development. Proc Natl Acad Sci USA. 2013;110(18):7312-7317.

14. Ludwig N, Yerneni SS, Razzo BM, Whiteside TL. Exosomes from HNSCC Promote Angiogenesis through Reprogramming of 
Endothelial Cells. Mol Cancer Res. 2018;16(11):1798-1808.

15. Carrasco-Ramírez P, et al. Podoplanin is a component of extracellular vesicles that reprograms cell-derived exosomal proteins and modulates lymphatic vessel formation. Oncotarget. 2016;7(13):16070-16089.

16. Li M, et al. Horizontal transfer of exosomal CXCR4 promotes murine hepatocarcinoma cell migration, invasion and lymphangiogenesis. Gene. 2018;676:101-109.

17. Umezu T, Ohyashiki K, Kuroda M, Ohyashiki JH. Leukemia cell to endothelial cell communication via exosomal miRNAs. Oncogene. 2013;32(22):2747-2755.

18. Umezu T, Tadokoro H, Azuma K, Yoshizawa S, Ohyashiki K, Ohyashiki JH. Exosomal miR-135b shed from hypoxic multiple myeloma cells enhances angiogenesis by targeting factor-inhibiting HIF-1. Blood. 2014;124(25):3748-3757.

19. Horie K, et al. Exosomes expressing carbonic anhydrase 9 promote angiogenesis. Biochem Biophys Res Commun. 2017;492(3):356-361.

20. Blomme A, et al. Myoferlin is a novel exosomal protein and functional regulator of cancer-derived exosomes. Oncotarget. 2016;7(50):83669-83683.

21. Tufail A, et al. Bevacizumab for neovascular age related macular degeneration (ABC Trial): multicentre randomised double masked study. BMJ. 2010;340:c2459.

22. Tewari KS, et al. Bevacizumab for advanced cervical cancer: final overall survival and adverse event analysis of a randomised, controlled, open-label, phase 3 trial (Gynecologic Oncology Group 240). Lancet. 2017;390(10103):1654-1663.

23. Perren TJ, et al. A phase 3 trial of bevacizumab in ovarian cancer. $N$ Engl J Med. 2011;365(26):2484-2496.

24. Miller K, et al. Paclitaxel plus bevacizumab versus paclitaxel alone for metastatic breast cancer. $N$ Engl J Med. 2007;357(26):2666-2676.

25. Bennouna J, et al. Continuation of bevacizumab after first progression in metastatic colorectal cancer (ML18147): a randomised phase 3 trial. Lancet Oncol. 2013;14(1):29-37.

26. Cunningham D, et al. Peri-operative chemotherapy with or without bevacizumab in operable oesophagogastric adenocarcinoma (UK Medical Research Council ST03): primary analysis results of a multicentre, open-label, randomised phase 2-3 trial. Lancet Oncol. 2017;18(3):357-370.

27. Roviello G, et al. No Advantage in Survival With Targeted Therapies as Maintenance in Patients With Limited and Extensive-Stage Small Cell Lung Cancer: A Literature-Based Meta-Analysis of Randomized Trials. Clin Lung Cancer. 2016;17(5):334-340.

28. Munk MR, Ceklic L, Ebneter A, Huf W, Wolf S, Zinkernagel MS. Macular atrophy in patients with long-term anti-VEGF treatment for neovascular age-related macular degeneration. Acta Ophthalmol. 2016;94(8):e757-e764.

29. Li K, Wong DK, Hong KY, Raffai RL. Cushioned-Density Gradient Ultracentrifugation (C-DGUC): A Refined and High Performance Method for the Isolation, Characterization, and Use of Exosomes. Methods Mol Biol. 2018;1740:69-83.

30. Théry C, et al. Minimal information for studies of extracellular vesicles 2018 (MISEV2018): a position statement of the International Society for Extracellular Vesicles and update of the MISEV2014 guidelines. J Extracell Vesicles. 2018;7(1):1535750.

31. Sinha S, et al. Cortactin promotes exosome secretion by controlling branched actin dynamics. J Cell Biol. 2016;214(2):197-213

32. Wang Y, et al. Ephrin-B2 controls VEGF-induced angiogenesis and lymphangiogenesis. Nature. 2010;465(7297):483-486.

33. Abéngozar MA, et al. Blocking ephrinB2 with highly specific antibodies inhibits angiogenesis, lymphangiogenesis, and tumor growth. Blood. 2012;119(19):4565-4576.

34. Adams RH, et al. Roles of ephrinB ligands and EphB receptors in cardiovascular development: demarcation of arterial/venous domains, vascular morphogenesis, and sprouting angiogenesis. Genes Dev. 1999;13(3):295-306.

35. Liu H, Devraj K, Möller K, Liebner S, Hecker M, Korff T. EphrinB-mediated reverse signalling controls junctional integrity and pro-inflammatory differentiation of endothelial cells. Thromb Haemost. 2014;112(1):151-163.

36. Landen CN, et al. Efficacy and antivascular effects of EphA2 reduction with an agonistic antibody in ovarian cancer. $J$ Natl Cancer Inst. 2006;98(21):1558-1570.

37. Sáinz-Jaspeado M, et al. EphA2-induced angiogenesis in ewing sarcoma cells works through bFGF production and is dependent on caveolin-1. PLoS ONE. 2013;8(8):e71449.

38. Youngblood V, et al. Elevated Slit2 Activity Impairs VEGF-Induced Angiogenesis and Tumor Neovascularization in EphA2-Deficient Endothelium. Mol Cancer Res. 2015;13(3):524-537.

39. Herbert SP, et al. Arterial-venous segregation by selective cell sprouting: an alternative mode of blood vessel formation. Science. 2009;326(5950):294-298.

40. Kaenel P, Hahnewald S, Wotzkow C, Strange R, Andres AC. Overexpression of EphB4 in the mammary epithelium shifts the differentiation pathway of progenitor cells and promotes branching activity and vascularization. Dev Growth Differ. 2014;56(4):255-275.

41. Groppa E, et al. EphrinB2/EphB4 signaling regulates non-sprouting angiogenesis by VEGF. EMBO Rep. 2018;19(5):e45054

42. Li DY, et al. Defective angiogenesis in mice lacking endoglin. Science. 1999;284(5419):1534-1537.

43. Paauwe M, et al. Endoglin targeting inhibits tumor angiogenesis and metastatic spread in breast cancer. Oncogene. 2016;35(31):4069-4079.

44. Han KY, Chang JH, Lee H, Azar DT. Proangiogenic Interactions of Vascular Endothelial MMP14 With VEGF Receptor 1 in VEGFA-Mediated Corneal Angiogenesis. Invest Ophthalmol Vis Sci. 2016;57(7):3313-3322.

45. Kajiya K, Hirakawa S, Ma B, Drinnenberg I, Detmar M. Hepatocyte growth factor promotes lymphatic vessel formation and function. EMBO J. 2005;24(16):2885-2895.

46. You WK, et al. VEGF and c-Met blockade amplify angiogenesis inhibition in pancreatic islet cancer. Cancer Res. 2011;71(14):4758-4768.

47. Beets $\mathrm{K}$, et al. BMP-SMAD signalling output is highly regionalized in cardiovascular and lymphatic endothelial networks. $B M C$ Dev Biol. 2016;16(1):34.

48. Weng J, et al. Activation of CD137 Signaling Promotes Angiogenesis in Atherosclerosis via Modulating Endothelial Smad1/5NFATc1 Pathway. J Am Heart Assoc. 2017;6(3):e004756.

49. Cheranov SY, Karpurapu M, Wang D, Zhang B, Venema RC, Rao GN. An essential role for SRC-activated STAT-3 in 14,15-EET-induced VEGF expression and angiogenesis. Blood. 2008;111(12):5581-5591. 
50. Gordon EJ, et al. The endothelial adaptor molecule TSAd is required for VEGF-induced angiogenic sprouting through junctional c-Src activation. Sci Signal. 2016;9(437):ra72.

51. Onishi M, et al. Annexin A2 regulates angiogenesis and invasion phenotypes of malignant glioma. Brain Tumor Pathol. 2015;32(3):184-194.

52. Fahmy K, et al. Myoferlin plays a key role in VEGFA secretion and impacts tumor-associated angiogenesis in human pancreas cancer. Int J Cancer. 2016;138(3):652-663.

53. Dong LD, et al. GluA2 trafficking is involved in apoptosis of retinal ganglion cells induced by activation of EphB/EphrinB reverse signaling in a rat chronic ocular hypertension model. J Neurosci. 2015;35(13):5409-5421.

54. Georgakopoulos A, Xu J, Xu C, Mauger G, Barthet G, Robakis NK. Presenilin1/gamma-secretase promotes the EphB2-induced phosphorylation of ephrinB2 by regulating phosphoprotein associated with glycosphingolipid-enriched microdomains/ Csk binding protein. FASEB J. 2011;25(10):3594-3604.

55. Sawamiphak S, et al. Ephrin-B2 regulates VEGFR2 function in developmental and tumour angiogenesis. Nature. 2010;465(7297):487-491.

56. Farshchian M, et al. EphB2 Promotes Progression of Cutaneous Squamous Cell Carcinoma. J Invest Dermatol. 2015;135(7):1882-1892.

57. Gibcus JH, et al. Cortactin expression predicts poor survival in laryngeal carcinoma. Br J Cancer. 2008;98(5):950-955.

58. Rodrigo JP, García LA, Ramos S, Lazo PS, Suárez C. EMS1 gene amplification correlates with poor prognosis in squamous cell carcinomas of the head and neck. Clin Cancer Res. 2000;6(8):3177-3182.

59. Ramos-García P, et al. Relevance of chromosomal band 11q13 in oral carcinogenesis: An update of current knowledge. Oral Oncol. 2017;72:7-16.

60. Coltrini D, Di Salle E, Ronca R, Belleri M, Testini C, Presta M. Matrigel plug assay: evaluation of the angiogenic response by reverse transcription-quantitative PCR. Angiogenesis. 2013;16(2):469-477.

61. Gonzalez-King H, García NA, Ontoria-Oviedo I, Ciria M, Montero JA, Sepúlveda P. Hypoxia Inducible Factor-1 $\alpha$ Potentiates Jagged 1-Mediated Angiogenesis by Mesenchymal Stem Cell-Derived Exosomes. Stem Cells. 2017;35(7):1747-1759.

62. Sahoo S, et al. Exosomes from human CD34(+) stem cells mediate their proangiogenic paracrine activity. Circ Res. 2011;109(7):724-728

63. Yang H, et al. Exosome-Derived miR-130a Activates Angiogenesis in Gastric Cancer by Targeting C-MYB in Vascular Endothelial Cells. Mol Ther. 2018;26(10):2466-2475.

64. Hui AB, et al. Comprehensive MicroRNA profiling for head and neck squamous cell carcinomas. Clin Cancer Res. 2010;16(4):1129-1139.

65. Lubov J, et al. Meta-analysis of microRNAs expression in head and neck cancer: uncovering association with outcome and mechanisms. Oncotarget. 2017;8(33):55511-55524.

66. Kong W, et al. Upregulation of miRNA-155 promotes tumour angiogenesis by targeting VHL and is associated with poor prognosis and triple-negative breast cancer. Oncogene. 2014;33(6):679-689.

67. Liu Y, et al. STAT3-regulated exosomal miR-21 promotes angiogenesis and is involved in neoplastic processes of transformed human bronchial epithelial cells. Cancer Lett. 2016;370(1):125-135.

68. Salvucci O, et al. EphrinB reverse signaling contributes to endothelial and mural cell assembly into vascular structures. Blood 2009;114(8):1707-1716.

69. Schust J, Sperl B, Hollis A, Mayer TU, Berg T. Stattic: a small-molecule inhibitor of STAT3 activation and dimerization. Chem Biol. 2006;13(11):1235-1242.

70. Ribeiro MF, Zhu H, Millard RW, Fan GC. Exosomes Function in Pro- and Anti-Angiogenesis. Curr Angiogenes. 2013;2(1):54-59.

71. Todorova D, Simoncini S, Lacroix R, Sabatier F, Dignat-George F. Extracellular Vesicles in Angiogenesis. Circ Res. 2017;120(10):1658-1673.

72. McBride JD, Rodriguez-Menocal L, Guzman W, Candanedo A, Garcia-Contreras M, Badiavas EV. Bone Marrow Mesenchymal Stem Cell-Derived CD63 ${ }^{+}$Exosomes Transport Wnt3a Exteriorly and Enhance Dermal Fibroblast Proliferation, Migration, and Angiogenesis In Vitro. Stem Cells Dev. 2017;26(19):1384-1398.

73. Shabbir A, Cox A, Rodriguez-Menocal L, Salgado M, Van Badiavas E. Mesenchymal Stem Cell Exosomes Induce Proliferation and Migration of Normal and Chronic Wound Fibroblasts, and Enhance Angiogenesis In Vitro. Stem Cells Dev. 2015;24(14):1635-1647.

74. Huang Z, Feng Y. Exosomes Derived From Hypoxic Colorectal Cancer Cells Promote Angiogenesis Through Wnt4-Induced $\beta$-Catenin Signaling in Endothelial Cells. Oncol Res. 2017;25(5):651-661.

75. Treps L, Perret R, Edmond S, Ricard D, Gavard J. Glioblastoma stem-like cells secrete the pro-angiogenic VEGF-A factor in extracellular vesicles. J Extracell Vesicles. 2017;6(1):1359479.

76. Giusti I, et al. From glioblastoma to endothelial cells through extracellular vesicles: messages for angiogenesis. Tumour Biol. 2016;37(9):12743-12753.

77. Chuang JY, et al. WISP-1 a novel angiogenic regulator of the CCN family promotes oral squamous cell carcinoma angiogenesis through VEGF-A expression. Oncotarget. 2015;6(6):4239-4252.

78. Oh SH, et al. Insulin-like growth factor binding protein-3 suppresses vascular endothelial growth factor expression and tumor angiogenesis in head and neck squamous cell carcinoma. Cancer Sci. 2012;103(7):1259-1266.

79. Becker A, Thakur BK, Weiss JM, Kim HS, Peinado H, Lyden D. Extracellular Vesicles in Cancer: Cell-to-Cell Mediators of Metastasis. Cancer Cell. 2016;30(6):836-848.

80. . Unified nomenclature for Eph family receptors their ligands, the ephrins. Eph Nomenclature Committee. Cell. 1997;90(3):403404.

81. Henkemeyer M, et al. Nuk controls pathfinding of commissural axons in the mammalian central nervous system. Cell. 1996;86(1):35-46.

82. Wilkinson DG. Multiple roles of EPH receptors and ephrins in neural development. Nat Rev Neurosci. 2001;2(3):155-164.

83. Park I, Lee HS. EphB/ephrinB signaling in cell adhesion and migration. Mol Cells. 2015;38(1):14-19.

84. Funk SD, Orr AW. Ephs and ephrins resurface in inflammation, immunity, and atherosclerosis. Pharmacol Res. 2013;67(1):42-52. 
85. Gong J, Körner R, Gaitanos L, Klein R. Exosomes mediate cell contact-independent ephrin-Eph signaling during axon guidance. J Cell Biol. 2016;214(1):35-44.

86. Luo H, et al. Efnb1 and Efnb2 proteins regulate thymocyte development, peripheral T cell differentiation, and antiviral immune responses and are essential for interleukin-6 (IL-6) signaling. J Biol Chem. 2011;286(48):41135-41152.

87. Stimamiglio MA, et al. EphB2-mediated interactions are essential for proper migration of T cell progenitors during fetal thymus colonization. J Leukoc Biol. 2010;88(3):483-494.

88. Bhatia S, et al. Inhibition of EphB4-Ephrin-B2 Signaling Reprograms the Tumor Immune Microenvironment in Head and Neck Cancers. Cancer Res. 2019;79(10):2722-2735.

89. Shiuan E, Chen J. Eph Receptor Tyrosine Kinases in Tumor Immunity. Cancer Res. 2016;76(22):6452-6457.

90. Zhao C, et al. Overexpression of junctional adhesion molecule-A and EphB2 predicts poor survival in lung adenocarcinoma patients. Tumour Biol. 2017;39(2):1010428317691000.

91. Lu Z, et al. Overexpression of the B-type Eph and ephrin genes correlates with progression and pain in human pancreatic cancer. Oncol Lett. 2012;3(6):1207-1212.

92. Hurwitz H, et al. Bevacizumab plus irinotecan, fluorouracil, and leucovorin for metastatic colorectal cancer. $N$ Engl J Med. 2004;350(23):2335-2342.

93. Robert NJ, et al. RIBBON-1: randomized, double-blind, placebo-controlled, phase III trial of chemotherapy with or without bevacizumab for first-line treatment of human epidermal growth factor receptor 2-negative, locally recurrent or metastatic breast cancer. J Clin Oncol. 2011;29(10):1252-1260.

94. Wick W, et al. Lomustine and Bevacizumab in Progressive Glioblastoma. N Engl J Med. 2017;377(20):1954-1963.

95. Voss MH, et al. Phase II Trial and Correlative Genomic Analysis of Everolimus Plus Bevacizumab in Advanced Non-Clear Cell Renal Cell Carcinoma. J Clin Oncol. 2016;34(32):3846-3853.

96. Socinski MA, et al. Atezolizumab for First-Line Treatment of Metastatic Nonsquamous NSCLC. N Engl J Med 2018;378(24):2288-2301.

97. Burger RA, et al. Incorporation of bevacizumab in the primary treatment of ovarian cancer. NEngl J Med. 2011;365(26):2473-2483.

98. Hensley ML, et al. Randomized phase III trial of gemcitabine plus docetaxel plus bevacizumab or placebo as first-line treatment for metastatic uterine leiomyosarcoma: an NRG Oncology/Gynecologic Oncology Group study. J Clin Oncol. 2015;33(10):1180-1185.

99. Machiels JP, et al. Phase II study of sunitinib in recurrent or metastatic squamous cell carcinoma of the head and neck: GORTEC 2006-01. J Clin Oncol. 2010;28(1):21-28.

100. Cohen EE, et al. Erlotinib and bevacizumab in patients with recurrent or metastatic squamous-cell carcinoma of the head and neck: a phase I/II study. Lancet Oncol. 2009;10(3):247-257.

101. Argiris A, et al. Phase II trial of pemetrexed and bevacizumab in patients with recurrent or metastatic head and neck cancer. J Clin Oncol. 2011;29(9):1140-1145.

102. Argiris A, et al. Cetuximab and bevacizumab: preclinical data and phase II trial in recurrent or metastatic squamous cell carcinoma of the head and neck. Ann Oncol. 2013;24(1):220-225.

103. Argiris A, et al. Phase II randomized trial of radiation therapy, cetuximab, and pemetrexed with or without bevacizumab in patients with locally advanced head and neck cancer. Ann Oncol. 2016;27(8):1594-1600.

104. Kozakiewicz P, Grzybowska-Szatkowska L. Application of molecular targeted therapies in the treatment of head and neck squamous cell carcinoma. Oncol Lett. 2018;15(5):7497-7505.

105. Athanassios Argiris SL, et al. Phase III randomized trial of chemotherapy with or without bevacizumab (B) in patients (pts) with recurrent or metastatic squamous cell carcinoma of the head and neck (R/M SCCHN): Survival analysis of E1305, an ECOG-ACRIN Cancer Research Group trial. J Clin Oncol. 2017;35(no. 15_suppl):6000.

106. Colevas AD, et al. NCCN Guidelines Insights: Head and Neck Cancers, Version 1.2018. J Natl Compr Canc Netw. 2018;16(5):479-490.

107. Montag M, et al. Angiogenic growth factors in tissue homogenates of HNSCC: expression pattern, prognostic relevance, and interrelationships. Cancer Sci. 2009;100(7):1210-1218.

108. Martone T, et al. Prognostic relevance of CD105+ microvessel density in HNSCC patient outcome. Oral Oncol. 2005;41(2):147-155.

109. Sano D, et al. Vandetanib restores head and neck squamous cell carcinoma cells' sensitivity to cisplatin and radiation in vivo and in vitro. Clin Cancer Res. 2011;17(7):1815-1827.

110. Myers JN, Holsinger FC, Jasser SA, Bekele BN, Fidler IJ. An orthotopic nude mouse model of oral tongue squamous cell carcinoma. Clin Cancer Res. 2002;8(1):293-298.

111. Aper SJ, et al. Colorful protein-based fluorescent probes for collagen imaging. PLoS ONE. 2014;9(12):e114983.

112. MacCoss MJ, et al. Shotgun identification of protein modifications from protein complexes and lens tissue. Proc Natl Acad Sci USA. 2002;99(12):7900-7905.

113. Martinez MN, et al. Obesity and altered glucose metabolism impact HDL composition in CETP transgenic mice: a role for ovarian hormones. J Lipid Res. 2012;53(3):379-389.

114. Yates JR, Eng JK, McCormack AL, Schieltz D. Method to correlate tandem mass spectra of modified peptides to amino acid sequences in the protein database. Anal Chem. 1995;67(8):1426-1436.

115. Vasaikar SV, Straub P, Wang J, Zhang B. LinkedOmics: analyzing multi-omics data within and across 32 cancer types. Nucleic Acids Res. 2018;46(D1):D956-D963.

116. Liberzon A, Birger C, Thorvaldsdóttir H, Ghandi M, Mesirov JP, Tamayo P. The Molecular Signatures Database (MSigDB) hallmark gene set collection. Cell Syst. 2015;1(6):417-425. 